\title{
Actions on Invariant Spheres around Isolated Fixed Points of Actions of Cyclic Groups
}

By

\author{
Masayoshi KAMATA*
}

\section{$\S 1$. Introduction}

Fix a prime number $p$ and let $Z_{p}$ be a cyclic group of order $p$. We consider a pair $(M, \phi)$ consisting of a compact simply connected almost complex manifold $M$ without boundary and a smooth $Z_{p}$-action $\phi$ : $Z_{p} \times M \rightarrow M$ preserving the almost complex structure of $M$. We suppose that $M$ is given an invariant Riemannian metric. If $a(\in M)$ is an isolated fixed point, then the induced action of $Z_{p}$ on the tangent space at $a$ gives a complex $Z_{p}$-module $V_{a}$ which has no trivial irreducible factor. Let $\xi$ : $E Z_{p} \rightarrow B Z_{p}$ be a universal principal $Z_{p}$-bundle and let $\xi\left(V_{a}\right): E Z_{p} \times_{z_{p}} V_{a}$ $\rightarrow B Z_{p}$ be the $V_{a}$-bundle associated with $\xi$. If $a$ and $b$ are isolated fixed points, we compare the cobordism Euler classes $e\left(\xi\left(V_{a}\right)\right)$ and $e\left(\xi\left(V_{b}\right)\right)$ which belong to the complex cobordism group $M U^{*}\left(B Z_{p}\right)$ of the classifying space $B Z_{p}$ of $Z_{p}$. Let $F_{U}$ be the universal formal group law over $M U^{*}$, and write

$$
x+{ }_{F} y=F_{U}(x, y) .
$$

For a positive integer $n,[n]_{F}(x)$ is inductively defined by

$$
[1]_{F}(x)=x
$$

and

$$
[n]_{F}(x)=[n-1]_{F}(x)+_{F} x .
$$

It is known that the cobordism ring $M U^{*}\left(B Z_{p}\right)$ is formal power series algebra $M U^{*}[[x]]$ over $M U^{*}$ modulo an ideal generated by $[p]_{F}(x)$

Communicated by N. Shimada, August 3, 1981.

* Department of Mathematics, College of General Education, Kyushu University, Fukuoka 810, Japan. 
[15]. Let us write

$$
[p]_{F}(x)=p x+a_{1}^{(p)} x^{2}+a_{2}^{(p)} x^{3}+\cdots,
$$

where $a_{i}^{(p)} \in M U^{-2 i}$, and

$$
\langle p\rangle_{F}(x)=p+a_{1}^{(p)} x+a_{2}^{(p)} x^{2}+\cdots .
$$

Let $S$ denote the multiplicative set in $M U^{*}\left(B Z_{p}\right)$ consisting of cobordism Euler classes $e(\xi(V)), V$ the non trivial complex $Z_{p}$-module, and let $\lambda$ : $M U^{*}\left(B Z_{p}\right) \rightarrow S^{-1} M U^{*}\left(B Z_{p}\right)$ be the canonical map [9]. In this paper we show the following

Theorem A. Assume that $H^{i}\left(B Z_{p} ;\left\{\pi_{i}(M)\right\}\right) \cong 0$ for $1 \leqq i \leqq 2 n$ -1 (cf. [4, p. 355]), and $\lambda(\alpha)=e\left(\xi\left(V_{a}\right)\right) / e\left(\xi\left(V_{b}\right)\right)$. Then for any Landweber-Novikov operation $S_{\omega}^{U}, \omega \neq(0)$ [14], [17], $S_{\omega}^{U}(\alpha)$ belongs to an ideal generated by $x^{n}$ and $\langle p\rangle_{F}(x)$ in $M U^{*}\left(B Z_{p}\right)$, where $x=$ $e(\xi(L))$ and $L$ is the canonical one dimensional complex $Z_{p}$-module with an action of $Z_{p}$ given by multiplication by $\rho=\exp (2 \pi i / p)$ on $C^{1}$.

The action of $Z_{p}$ on $M$ induces a natural action on a unit sphere $S\left(V_{a}\right)$ in a tangent space $V_{a}$ at an isolated fixed point $a$ which is equivalent to the action of $Z_{p}$ on a sphere around the fixed point. The action $\phi_{a}: Z_{p} \times S\left(V_{a}\right) \rightarrow S\left(V_{a}\right)$ determines a weakly complex bordism class $\left[S\left(V_{a}\right), \phi_{a}\right]$ of the bordism group $M U_{*}\left(Z_{p}\right)$ of fixed point free $Z_{p}$ actions preserving a weakly complex structure, which is generated as an $M U_{*}$ module by the set of $Z_{p}$-manifolds $\left\{\left[S^{2 n+1}, \widetilde{\phi}\right]\right\}$, where the action $\widetilde{\phi}$ of $Z_{p}$ on a sphere $S^{2 n+1} \subset C^{n+1}$ is defined by $\widetilde{\phi}(g, z)=\rho z, g$ a generator of $Z_{p}$ [6], [11]. Kasparov in [13] showed that the weakly complex bordism class $\left[S\left(V_{a}\right), \phi_{a}\right]$ is computable. By making use the Kasparov theorem and Theorem $\mathrm{A}$, we obtain the following

Theorem B. Assume that $H^{i}\left(B Z_{p} ;\left\{\pi_{i}(M)\right\}\right) \cong 0$ for $1 \leqq i \leqq 2 n-1$. If $V_{a}=L^{l_{1}} \oplus \cdots \oplus L^{l_{k}}$ and $V_{b}=L^{m_{1}} \oplus \cdots \oplus L^{m_{k}}$, then

$$
\begin{aligned}
l_{1} \cdots l_{k} & {\left[S\left(V_{a}\right), \phi_{a}\right]-m_{1} \cdots m_{k}\left[S\left(V_{b}\right), \phi_{b}\right] } \\
= & \widetilde{\mu}_{1}\left[S^{2 k-3}, \widetilde{\phi}\right]+\widetilde{\mu}_{2}\left[S^{2 k-5}, \widetilde{\phi}\right]+\cdots+\widetilde{\mu}_{k-1}\left[S^{1}, \widetilde{\phi}\right]
\end{aligned}
$$

where $\tilde{\mu}_{1}, \widetilde{\mu}_{2}, \cdots, \widetilde{\mu}_{n-1}$ belong to an ideal generated by $p, a_{1}^{(p)}, a_{2}^{(p)}, \cdots$. 
$a_{l}^{(p)}, \cdots$ in $M U^{*}$.

In Section 2 we investigate $S^{1}$-actions on a product space $S^{2 n+1} \times$ $S^{2 n+1}$ of spheres and equivariant maps between the $S^{1}$-spaces. In Section 3 the Umkehr homomorphism of some map between the orbit spaces $\left(S^{2 n+1} \times S^{2 m+1}\right) / S^{1}$ is computed to give a slightly different proof of the Kasparov theorem [13] in Section 4. In Section 5 we discuss about relations among cobordism characteristic classes [7] of $\xi\left(V_{a}\right)$ and $\xi\left(V_{b}\right)$ and give a proof of Theorem A. Section 6 is devoted to prove Theorem B. In Section 7 we study the isolated fixed point set of $Z_{3}$-actions.

Bredon in Section 10 of Chapter VI of [4] compared representations at two fixed points of a smooth action, by using equivariant $K$-theory.

\section{$\S 2$. On Orbit Spaces of $S^{2 n+1} \times S^{2 n+1}$ with Respect to $S^{1}$}

We define $\phi\left(l_{0}, l_{1}, \cdots, l_{n}\right): S^{1} \times S^{2 m+1} \times S^{2 n+1} \rightarrow S^{2 m+1} \times S^{2 n+1}$ by

$$
\begin{array}{r}
\phi\left(l_{0}, l_{1}, \cdots, l_{n}\right)\left(z,\left(u_{0}, u_{1}, \cdots, u_{m}\right),\left(v_{0}, v_{1}, \cdots, v_{n}\right)\right) \\
=\left(\left(z u_{0}, z u_{1}, \cdots, z u_{m}\right),\left(z^{l_{0}} v_{0}, z^{l_{1}} v_{1}, \cdots, z^{l_{n}} v_{n}\right)\right) .
\end{array}
$$

This is differentiable and the orbit space $\left(S^{2 m+1} \times S^{2 n+1}\right) / \phi\left(l_{0}, \cdots, l_{n}\right)$ is an orientable smooth manifold. Let $S^{1}$ act on $S^{2 m+1} \times C^{1}$ by

$$
z \cdot\left(\left(u_{0}, \cdots, u_{m}\right), v\right)=\left(\left(\approx u_{0}, \cdots, z u_{m}\right), z v\right) .
$$

The orbit space induces a complex line bundle over the complex projective space

$$
\pi: S^{2 m+1} \times{ }_{S 1} C^{1} \rightarrow S^{2 m+1} / S^{1}=C P^{m}, \pi([u, v])=[u]
$$

which is denoted by $\eta$. The total space $S\left(\eta^{l_{0}} \oplus \cdots \oplus \eta^{l_{n}}\right)$ of the sphere bundle associated with $\eta^{l_{0}} \oplus \cdots \oplus \eta^{l_{n}}$ is diffeomorphic to $\left(S^{2 m+1} \times S^{2 n+1}\right) /$ $\phi\left(l_{0}, \cdots, l_{n}\right)$. The structure of the integral cohomology group $H^{*}\left(S\left(\eta^{l_{0}} \oplus\right.\right.$ $\left.\left.\cdots \oplus \eta^{l_{n}}\right)\right)$ is determined as follows in [18].

Proposition 2.1. (1) If $m \leqq n$, then $H^{2 j}\left(S\left(\eta^{l_{0}} \oplus \cdots \oplus \eta^{l_{n}}\right)\right) \cong$ $H^{2 j}\left(C P^{m}\right)$ and $H^{2 j-1}\left(S\left(\eta^{l_{0}} \oplus \cdots \oplus \eta^{l_{n}}\right)\right) \cong H^{2 j-2(n+1)}\left(C P^{m}\right)$.

(2) If $m>n$, then 


$$
\begin{aligned}
& H^{2 j}\left(S\left(\eta^{l_{0}} \oplus \cdots \oplus \eta^{l_{n}}\right)\right) \cong\left\{\begin{array}{l}
0, \quad j>m \\
Z /\left(l_{0} \cdots l_{n}\right), \quad n+1 \leqq j \leqq m \\
H^{2 j}\left(C P^{m}\right), \quad j \leqq n
\end{array}\right. \\
& H^{2 j-1}\left(S\left(\eta^{l_{0}} \oplus \cdots \oplus \eta^{l_{n}}\right)\right) \cong \begin{cases}0, & 0 \leqq j \leqq m \\
H^{2 j-2(n+1)}\left(C P^{m}\right), & m+1 \leqq j\end{cases}
\end{aligned}
$$

The map $f: S^{2 m+1} \times S^{2 n+1} \rightarrow S^{2 m+1} \times S^{2 n+1}$ defined by

$$
\begin{aligned}
& f\left(\left(u_{0}, \cdots, u_{m}\right),\left(v_{0}, \cdots, v_{n}\right)\right)=\left(\left(u_{0}, \cdots, u_{m}\right), \frac{1}{r}\left(v_{0}^{l_{0}}, \cdots, v_{n}^{l_{n}}\right)\right), \\
& r=\sqrt{\left|v_{0}\right|^{2 l_{0}}+\cdots+\left|v_{n}\right|^{2 l_{n}}},
\end{aligned}
$$

induces a map of the orbit spaces

$$
\tilde{f}:\left(S^{2 m+1} \times S^{2 n+1}\right) / \phi(1, \cdots, 1) \rightarrow\left(S^{2 m+1} \times S^{2 n+1}\right) / \phi\left(l_{0}, \cdots, l_{n}\right) .
$$

Denote by $[M]$ the fundamental class of a compact orientable manifold $M$. Then we have

Proposition 2. 2. $\tilde{f}_{*}\left[\left(S^{2 m+1} \times S^{2 n+1}\right) / \phi(1, \cdots, 1)\right]$

$$
=l_{0} l_{1} \cdots l_{n}\left[\left(S^{2 m+1} \times S^{2 n+1}\right) / \phi\left(l_{0}, \cdots, l_{n}\right)\right] .
$$

Proof. $\tilde{f}$ is a fiber preserving map of sphere bundles $S((n+1) \eta)$ and $S\left(\eta^{l_{0}} \oplus \cdots \oplus \eta^{l_{n}}\right)$, as $\eta^{l_{0}} \oplus \cdots \oplus \eta^{l_{n}}$ is isomorphic to a bundle of an orbit space of an $S^{1}$-action on $S^{2 m+1} \times C^{n+1}$ defined by

$$
z \cdot\left(u,\left(v_{0}, \cdots, v_{n}\right)\right)=\left(z u,\left(z^{l_{0}} v_{0}, \cdots, z^{l_{n}} v_{n}\right)\right) .
$$

Let $f_{1}$ be a fiber preserving map from $(n+1) \eta$ to $\eta^{l_{0}} \oplus \cdots \oplus \eta^{l_{n}}$ defined by

$$
f_{1}\left(u,\left(v_{0}, \cdots, v_{n}\right)\right)=\left(u,\left(v_{0}^{l_{0}}, \cdots, v_{n}^{l_{n}}\right)\right)
$$

which induces a map between the Thom complexes

$$
\tilde{f}_{1}: T(1, \cdots, 1) \rightarrow T\left(l_{0}, \cdots, l_{n}\right),
$$

where $T\left(l_{0}, \cdots, l_{n}\right)=E\left(l_{0}, \cdots, l_{n}\right) /\left\{E\left(l_{0}, \cdots, l_{n}\right)\right.$-the zero section $\}$, and $E\left(l_{0}\right.$, $\left.\cdots, l_{n}\right)$ is the total space of $\eta^{l_{0}} \oplus \cdots \oplus \eta^{l_{n}} . \quad S\left(\eta^{l_{0}} \oplus \cdots \oplus \eta^{l_{n}}\right)$ and $E\left(l_{0}, \cdots\right.$, $l_{n}$ )-\{the zero section $\}$ are of the same homotopy type, and the following diagram is homotopy commutative 


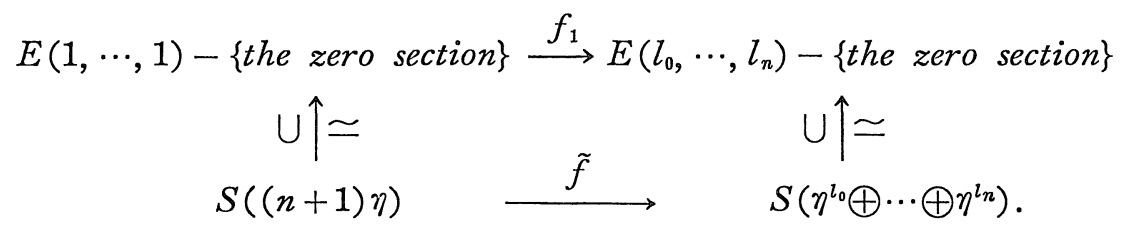

Let $t\left(l_{0}, \cdots, l_{n}\right)$ be the Thom class of $\eta^{l_{0}} \oplus \cdots \oplus \eta^{l_{n}}$. Then we have $\tilde{f}_{1}{ }^{*}\left(t\left(l_{0}, \cdots, l_{n}\right)\right)=l_{0} l_{1} \cdots l_{n} t(1, \cdots, 1)$. Since the coboundary homomorphism $\delta: H^{2 m+2 n+1}\left(S\left(\eta^{l_{0}} \oplus \cdots \oplus \eta^{l_{n}}\right)\right) \rightarrow \widetilde{H}^{2 m+2 n+2}\left(T\left(l_{0}, \cdots, l_{n}\right)\right)$ is isomorphic, the fundamental class of $\left(S^{2 m+1} \times S^{2 n+1}\right) / \phi\left(l_{0}, \cdots, l_{n}\right)$ is the dual class of $\delta^{-1}\left\{\pi^{*}\left(\left[C P^{m}\right]^{*}\right) \cup t\left(l_{0}, \cdots, l_{n}\right)\right\}$, where $\pi: E\left(l_{0}, \cdots, l_{n}\right) \rightarrow C P^{m}$ is the projection and $\left[C P^{m}\right]^{*}$ is the dual of $\left[C P^{m}\right]$. Then the assertion follows.

Suppose that $M^{m}$ and $N^{n}$ are orientable manifolds. A continuous map $h: M^{m} \rightarrow N^{n}$ determines the Umkehr homomorphism

$$
h_{!}: H^{k}\left(M^{m}\right) \stackrel{D}{\cong} H_{m-k}\left(M^{m}\right) \stackrel{h_{*}}{\longrightarrow} H_{m-k}\left(N^{n}\right) \stackrel{D^{-1}}{\cong} H^{n-m+k}\left(N^{n}\right)
$$

where $D$ is the Poincare duality.

Proposition 2.3. Assume that $g$ is an embedding of ( $S^{2 m+1}$ $\left.\times S^{2 n+1}\right) / \phi(1, \cdots, 1)$ into $S^{N}$ for a large $N$. Then the Umkehr homomorphism of

$F=\tilde{f} \times g:\left(S^{2 m+1} \times S^{2 n+1}\right) / \phi(1, \cdots, 1) \rightarrow\left(S^{2 m+1} \times S^{2 n+1}\right) / \phi\left(l_{0}, \cdots, l_{n}\right) \times S^{N}$, $\tilde{f} \times g(x)=(\tilde{f}(x), g(x))$, satisfies

$$
F_{!}\left(\tilde{f}^{*}(y)\right)=l_{0} \cdots l_{n} y \times\left[S^{N}\right]^{*}
$$

where $\left[S^{N}\right]^{*}$ is the dual of $\left[S^{N}\right]$.

Proof. The Umkehr homomorphism satisfies $F_{!}\left(F^{*}(a) \cup b\right)=a \cup$ $F_{!}(b)$ [8]. We calculate using Proposition 2.2,

$$
\begin{aligned}
F_{!}\left(\tilde{f}^{*}(y)\right) & \\
& =(y \times 1) \cup F_{!}(1) \\
& =(y \times 1) \cup D^{-1}(\tilde{f} \times g)_{*}\left[\left(S^{2 m+1} \times S^{2 n+1}\right) / \phi(1, \cdots, 1)\right] \\
& =(y \times 1) \cup D^{-1}\left(\left(l_{0} \cdots l_{n}\right)\left[\left(S^{2 m+1} \times S^{2 n+1}\right) / \phi\left(l_{0}, \cdots, l_{n}\right)\right] \times 1\right) \\
& =(y \times 1) \cup l_{0} \cdots l_{n}\left(1 \times\left[S^{N}\right]^{*}\right) .
\end{aligned}
$$


If $m \leqq n$, then we get a short exact sequence

$$
\begin{aligned}
0 \rightarrow M U^{*}\left(C P^{m}\right) & \stackrel{\pi^{*}}{\rightarrow} M U^{*}\left(S\left(\eta^{l_{0}} \oplus \cdots \oplus \eta^{l_{n}}\right)\right) \\
& \stackrel{\delta}{\rightarrow} \widetilde{M U}\left(T\left(l_{0}, \cdots, l_{n}\right)\right) \rightarrow 0
\end{aligned}
$$

and $\delta: M U^{2 n+1}\left(S\left(\eta^{l_{0}} \oplus \cdots \oplus \eta^{l_{n}}\right)\right) \rightarrow \widetilde{M U}^{2 n+2}\left(T\left(l_{0}, \cdots, l_{n}\right)\right) \quad$ is isomorphic. In this case we may determine the ring structure of $M U^{*}\left(\left(S^{2 m+1} \times S^{2 n+1}\right) /\right.$ $\left.\phi\left(l_{0}, \cdots, l_{n}\right)\right)$ (cf. [18]).

Proposition 2.4. If $m \leqq n$, then $M U^{*}\left(\left(S^{2 m+1} \times S^{2 n+1}\right) / \phi\left(l_{0}, \cdots, l_{n}\right)\right)$ is $M U^{*}[x, y] /\left(x^{m+1}, y^{2}\right)$ where $x$ is the first cobordism Chern class $c_{U}^{1}\left(\pi^{\prime} \eta\right)$ and $y$ is an element of $M U^{2 n+1}\left(S\left(\eta^{l_{0}} \oplus \cdots \oplus \eta^{l_{n}}\right)\right)$ such that $\delta y$ is the Thom class of $\eta^{l_{0}} \oplus \cdots \oplus \eta^{l_{n}}$.

Proof. $M U^{*}\left(S\left(\eta^{l_{0}} \oplus \cdots \oplus \eta^{l_{n}}\right)\right)$ is isomorphic to the direct sum of $M U^{*}\left(C P^{m}\right)$ and $\widetilde{M U}^{*}\left(T\left(l_{0}, \cdots, l_{n}\right)\right)$. We have

$$
(-1)^{\operatorname{deg} a} \delta\left(\pi^{*} a \bigcup b\right)=\pi^{*} a \bigcup \delta b
$$

(cf. Chapter 13 of $[20])$, and $M U^{*}\left(S\left(\eta^{l_{0}} \oplus \cdots \oplus \eta^{l_{n}}\right)\right.$ ) is a free $M U^{*}$. module generated by $\left\{\left(\pi^{*} x\right)^{i}, i=1,2, \cdots, m\right\}$ and $\left\{\left(\pi^{*} x\right)^{i} \cup y, i=1,2\right.$, $\cdots, m\}$. It follows from Proposition 2.1 that $M U^{2(2 n+1)}\left(S\left(\eta^{l_{0}} \oplus \cdots \oplus \eta^{l_{n}}\right)\right)$ is zero.

Q.E.D.

\section{§ 3. On the Umkehr Homomorphism of $\tilde{f}$ with the $M U^{*}$.Orientation}

For any set $\omega=\left(i_{1}, \cdots, i_{r}\right)$ of positive integers, let $\sum t_{1}^{i_{1}} \cdots t_{r}^{i_{r}}$ be the symmetric polynomial of variable $t_{j}, 1 \leqq j \leqq n$ to be the smallest symmetric polynomial containing the monomial $t_{1}^{i_{1}} \cdots t_{r}^{i_{r}}$, which is expressible uniquely as a polynomial with integral coefficients in the elementary symmetric polynomials $\mathfrak{S}_{1}, \mathfrak{S}_{2}, \cdots, \mathfrak{S}_{n}$ of the $t$ 's and write

$$
P_{\omega}\left(\mathfrak{S}_{1}, \mathfrak{\Im}_{2}, \cdots, \mathfrak{\Im}_{n}\right)=\sum t_{1}^{i_{1}} \cdots t_{r}^{i_{r}} \text {. }
$$

For an $n$-dimensional complex vector bundle $\zeta$ over $X$, we define

$$
c_{\omega}^{H}(\zeta)=P_{\omega}\left(c_{H}^{1}(\zeta), c_{H}^{2}(\zeta), \cdots, c_{H}^{n}(\zeta)\right)
$$

and $c_{(0, \ldots, 0)}^{H}(\zeta)=1$, where $c_{H}^{i}(\zeta)$ are the ordinary cohomology Chern classes. 
Suppose that $x \in M U^{k}(X)$ is represented by

$$
g: S^{2 N-k} X^{+} \rightarrow M U(N) .
$$

We define

$$
S_{\omega}^{H}(x)=\sigma^{k-2 N} g^{*} \Phi c_{\omega}^{H}\left(\gamma_{N}\right),
$$

where $\Phi: H^{*}(B U(N)) \rightarrow \widetilde{H}^{*}(M U(N))$ is the Thom isomorphism, $\sigma^{k-2 N}$ denotes $(k-2 N)$-fold iterated suspension isomorphism and $\gamma_{N}$ is the $N$. dimensional universal complex vector bundle. The ring $H_{*}(M U)$ is isomorphic to $Z\left[t_{1}, t_{2}, \cdots\right]$. Let

$$
\omega=(\underbrace{1, \cdots, 1}_{i_{1}}, \underbrace{2, \cdots, 2}_{i_{2}}, \cdots, \underbrace{k, \cdots, k}_{i_{k}})
$$

and we define

$$
|\omega|=i_{1}+2 i_{2}+\cdots+k i_{k}
$$

and

$$
t^{\omega}=t_{1}^{i_{1}} t_{2}^{i_{2}} \cdots t_{k}^{i_{k}}
$$

There exists a multiplicative natural transformation

$$
\beta_{H}: M U^{*}(X) \rightarrow(H \wedge M U)^{*}(X)=H^{*}(X)\left[\left[t_{1}, t_{2}, \cdots\right]\right]
$$

defined by

$$
\beta_{H}(x)=\sum_{\omega} s_{\omega}^{H}(x) t^{\omega}
$$

which is called Boardman map (cf. [1]). $\beta_{H}: M U^{*}\left(S^{0}\right) \rightarrow H_{*}(M U)$ is the Hurewicz homomorphism which is injective [16]. Given $x \in M U^{*}(X)$ with $x=\left[g: S^{2 N-k} X^{+} \rightarrow M U(N)\right]$, the Thom homomorphism $\mu: M U^{k}(X)$ $\rightarrow H^{k}(X)$ is defined by $\mu(x)=\sigma^{k-2 N} g^{*} \Phi(1)=S_{(0, \ldots, 0)}^{H}(x)$.

Proposition 3.1. Suppose that a finite $C W$-complex $X$ has no torsion in its integral cohomology, then the Boardman map $\beta_{H}$ is injective.

Proof. Since the cohomology of $X$ has no torsion, the Thom homomorphism is surjective. Suppose that $y_{1}^{(n)}, y_{2}^{(n)}, \cdots, y_{i_{n}}^{(n)}$ are the basis of $H^{n}(X)$, then we can take $u_{j}^{(n)}$ with $\mu\left(u_{j}^{(n)}\right)=y_{j}^{(n)}$. The correspondence 
$\sum y_{j}^{(n)} \otimes b_{j}^{(n)} \rightarrow \sum b_{j}^{(n)} u_{j}^{(n)}$ yields an isomorphism $H^{*}(X) \otimes M U^{*} \cong M U^{*}(X)$ (cf. [5]). We see

$$
\beta_{H}\left(\sum b_{j}^{(n)} u_{j}^{(n)}\right)=\sum \beta_{H}\left(b_{j}^{(n)}\right)\left\{y_{j}^{(n)}+\sum_{|\omega|>0} S_{\omega}^{H}\left(u_{j}^{(n)}\right) t^{\omega}\right\} .
$$

Let $\beta_{H}\left(\sum b_{j}^{(n)} u_{j}^{(n)}\right)=0$, and we can derive inductively that $\beta_{H}\left(b_{j}^{(n)}\right)=0$ and $b_{j}^{(n)}=0$.

For an $n$-dimensional complex vector bundle $\zeta$ over $X$, consider a formal power series of $t$ 's:

$$
c_{t}^{H}(\zeta)=\sum_{\omega} c_{\omega}^{H}(\zeta) t^{\omega} .
$$

This satisfies the naturality and $c_{t}^{H}\left(\zeta_{1} \oplus \zeta_{2}\right)=c_{t}^{H}\left(\zeta_{1}\right) c_{t}^{H}\left(\zeta_{2}\right)$. Suppose that $X$ and $M$ are weakly almost complex manifolds. An embedding $h: M$ $\rightarrow X$ with the normal vector bundle $\nu$ equipped with the complex structure induces the Umkehr homomorphisms:

$$
h_{1}: M U^{*}(M) \rightarrow M U^{*}(X),
$$

and

$$
h_{!}^{H}: H^{*}(M)\left[\left[t_{1}, t_{2}, \cdots\right]\right] \rightarrow H^{*}(X)\left[\left[t_{1}, t_{2}, \cdots\right]\right] .
$$

Now we recall the following (cf. [19])

Theorem 3. 2. $\beta_{H}\left(h_{!}(1)\right)=h_{!}^{H}\left(c_{t}^{H}(\nu)\right)$.

Proof. A composition of a collapsing map $c$ of the Thom construction and a classifying map $g_{\nu}$ for $\nu$

$$
\tilde{g}_{\nu}: X \stackrel{c}{\longrightarrow} T(\nu) \stackrel{g_{\nu}}{\longrightarrow} M U(k)
$$

represents $h_{1}(1) \in M U^{*}(X)$. By making use of the following commutative diagram:

$$
\begin{array}{ccc}
H_{*}(X) \stackrel{D}{\cong} H^{*}(X) \stackrel{c^{*}}{\longleftarrow} \widetilde{H}^{*}(T(\nu)) \\
\uparrow h_{*} & \stackrel{D}{\cong} & \cong \uparrow \Phi \\
H_{*}(M) & \stackrel{\uparrow}{\cong} & H^{*}(M)
\end{array}
$$


we calculate

$$
\begin{aligned}
\beta_{H}\left(h_{!}(1)\right) & =\sum_{\omega} S_{\omega}^{H}\left[g_{\nu} c\right] t^{\omega} \\
& =\sum_{\omega} c^{*} \Phi_{H} c_{\omega}^{H}(\nu) t^{\omega} \\
& =h_{!}^{H}\left(\sum_{\omega} c_{\omega}^{H}(\nu) t^{\omega}\right) .
\end{aligned}
$$

Q.E.D.

$M U^{*}(B U(1))$ is isomorphic to $M U^{*}\left[\left[x_{M U}\right]\right], x_{M U}=c_{U}^{1}\left(\gamma_{1}\right)$. The first cobordism Chern class $c_{U}^{1}\left(\gamma_{1}^{k}\right)$ of the $k$-fold tensor product of $\gamma_{1}$ is described as

$$
\begin{aligned}
c_{U}^{1}\left(\gamma_{1}^{k}\right) & =[k]_{F}\left(x_{M U}\right) \\
& =k x_{M U}+a_{1}^{(k)} x_{M U}^{2}+a_{2}^{(k)} x_{M U}^{3}+\cdots .
\end{aligned}
$$

Let $g: X \rightarrow B U(1)$ be a classifying map for a complex line bundle $\zeta$ over $X$. We see

$$
\langle k\rangle_{F}\left(c_{U}^{1}(\zeta)\right)=g^{*}\left\{k+a_{1}^{(k)} x_{M U}+a_{2}^{(k)} x_{M U}^{2}+\cdots\right\} .
$$

The map $\tilde{f}:\left(S^{2 m+1} \times S^{2 n+1}\right) / \phi(1, \cdots, 1) \rightarrow\left(S^{2 m+1} \times S^{2 n+1}\right) / \phi\left(l_{0}, \cdots, l_{n}\right)$ defined by

$$
\begin{aligned}
& \tilde{f}\left(\left[\left(u_{0}, \cdots, u_{m}\right),\left(v_{0}, \cdots, v_{n}\right)\right]\right. \\
& \quad=\left[\left(u_{0}, \cdots, u_{m}\right), \frac{1}{r}\left(v_{0}^{l_{0}}, \cdots, v_{n}^{l_{n}}\right)\right], \\
& r=\sqrt{\left|v_{0}\right|^{2 l_{0}}+\cdots+\left|v_{n}\right|^{2 l_{n}}},
\end{aligned}
$$

and an embedding $h:\left(S^{2 m+1} \times S^{2 n+1}\right) / \phi(1, \cdots, 1) \rightarrow S^{2 N}$ for a large $N$ determine a bordism class $\left[\left(S^{2 m+1} \times S^{2 n+1}\right) / \phi(1, \cdots, 1), \tilde{f} \times h\right]$ of $M U_{*}\left(\left(S^{2 m+1}\right.\right.$ $\left.\left.\times S^{2 n+1}\right) / \phi\left(l_{0}, \cdots, l_{n}\right) \times S^{2 N}\right)$. The projection $\pi:\left(S^{2 m+1} \times S^{2 n+1}\right) / \phi\left(l_{0}, \cdots, l_{n}\right)$ $\rightarrow C P^{m}$ is defined by $\pi[u, v]=[u]$. Then we have

Theorem 3.3. Suppose that $m \leqq n$. Then it follows that

$$
\begin{aligned}
& {\left[\left(S^{2 m+1} \times S^{2 n+1}\right) / \phi(1, \cdots, 1), \tilde{f} \times h\right]} \\
& \quad=D_{M U} \pi^{*}\left(\left\langle l_{0}\right\rangle_{F}\left(c_{U}^{1}(\eta)\right)\left\langle l_{1}\right\rangle_{F}\left(c_{U}^{1}(\eta)\right) \cdots\left\langle l_{n}\right\rangle_{F}\left(c_{U}^{1}(\eta)\right)\right) \times\left[P \subset S^{2 N}\right]
\end{aligned}
$$

where $P=\{a$ point $\}$ and $D_{M U}$ is the Atiyah-Poincare isomorphism [3]. 
Proof. If $m \leqq n$, then $H^{*}\left(\left(S^{2 m+1} \times S^{2 n+1}\right) / \phi\left(l_{0}, \cdots, l_{n}\right)\right)$ has no torsion from Propositions 2.1 and 3.1 implies that

$$
\begin{aligned}
\beta_{H}: M U^{*} & \left(\left(S^{2 m+1} \times S^{2 n+1}\right) / \phi\left(l_{0}, \cdots, l_{n}\right) \times S^{2 N}\right) \\
& \rightarrow H^{*}\left(\left(S^{2 m+1} \times S^{2 n+1}\right) / \phi\left(l_{0}, \cdots, l_{n}\right) \times S^{2 N}\right)\left[t_{1}, t_{2}, \cdots\right]
\end{aligned}
$$

is injective. The tangent bundle of $\left(S^{2 m+1} \times S^{2 n+1}\right) / \phi\left(l_{0}, \cdots, l_{n}\right)$ is stably isomorphic to $\pi^{!}\left(\tau\left(C P^{m}\right) \oplus \eta^{l_{0}} \oplus \cdots \oplus \eta^{l_{n}}\right)$ where $\eta$ is the Hopf bundle over $C P^{m}$ and $\tau(M)$ denotes the tangent bundle of $M[18]$. The normal vector bundle $\nu$ for $\tilde{f} \times h$ satisfies that $\nu \oplus \tau\left(\left(S^{2 m+1} \times S^{2 n+1}\right) / \phi(1, \cdots, 1)\right)$ is isomorphic to $\tilde{f}^{!} \tau\left(\left(S^{2 m+1} \times S^{2 n+1}\right) / \phi\left(l_{0}, \cdots, l_{n}\right)\right) \oplus 2 N \varepsilon$, where $\varepsilon$ is a trivial real line bundle. It follows directly from the definition that

$$
c_{t}^{H}(\eta)=1+x t_{1}+x^{2} t_{2}+\cdots+x^{m} t_{m}, \quad x=c_{H}^{1}(\eta)
$$

and

$$
c_{t}^{I I}(\nu)=\pi^{*}\left\{\frac{c_{t}^{H}\left(\eta^{l_{0}}\right) \cdots c_{t}^{H}\left(\eta^{l_{n}}\right)}{\left\{c_{t}^{H}(\eta)\right\}^{n+1}}\right\},
$$

since the following diagram is commutative

$$
\left(S^{2 m+1} \times S^{2 n+1}\right) / \phi(1, \cdots, 1) \stackrel{\tilde{f}}{\rightarrow}\left(S^{2 m+1} \times S^{2 n+1}\right) / \phi\left(l_{0}, \cdots, l_{n}\right)
$$

By using Theorem 3.2 and Proposition 2.3 we have

$$
\begin{aligned}
\beta_{H}\left((\tilde{f} \times h)_{!}(1)\right) & =(\tilde{f} \times h)_{!}^{H} c_{t}^{H}(\nu) \\
& =\pi^{*}\left\{\frac{l_{0} \cdots l_{n} c_{t}^{H}\left(\eta^{l_{0}}\right) \cdots c_{t}^{H}\left(\eta^{l_{n}}\right)}{\left\{c_{t}^{H}(\eta)\right\}^{n+1}}\right\} \times\left[S^{2 N}\right]^{*}
\end{aligned}
$$

On the other hand, we see that

$$
\beta_{H}\left(c_{U}^{1}\left(\eta^{k}\right)\right)=c_{H}^{1}\left(\eta^{k}\right) c_{t}^{H}\left(\eta^{k}\right)=k c_{H}^{1}(\eta) c_{t}^{H}\left(\eta^{k}\right)
$$

and

$$
\beta_{H}\left(c_{U}^{1}\left(\eta^{k}\right)\right)=\beta_{H}\left(\langle k\rangle_{F}\left(c_{U}^{1}(\eta)\right) \cdot c_{U}^{1}(\eta)\right)=\beta_{H}\left(\langle k\rangle_{F}\left(c_{U}^{1}(\eta)\right)\right) \beta_{H}\left(c_{U}^{1}(\eta)\right) .
$$

Therefore we have

$$
\beta_{H}\left(\langle k\rangle_{F}\left(c_{H}^{1}(\eta)\right)\right)=\frac{k c_{t}^{H}\left(\eta^{k}\right)}{c_{t}^{H}(\eta)} .
$$


Noting that $\beta_{H}$ maps $D_{M U}^{-1}\left(\left[P \subset S^{2 N}\right]\right)$ to $\left[S^{2 N}\right]^{*}$, we obtain

$$
\begin{gathered}
\beta_{H}\left(\pi^{*}\left\{\left\langle l_{0}\right\rangle_{F}\left(c_{U}^{1}(\eta)\right) \cdots\left\langle l_{n}\right\rangle_{F^{\prime}}\left(c_{U}^{1}(\eta)\right)\right\} \times D_{M U}^{-1}\left(\left[P \subset S^{2 N}\right]\right)\right. \\
=\beta_{H}\left((\tilde{f} \times h)_{!}(1)\right) .
\end{gathered}
$$

This completes the proof.

\section{$\S$ 4. Another Proof of the Kasparov Theorem}

Let $l_{0}, l_{1}, \cdots, l_{n}$ be integers prime to $p$. An action of $Z_{p}$ on $S^{2 m+1}$ $\times S^{2 n+1}$ is defined by

$$
\begin{array}{r}
\phi_{p}\left(l_{0}, \cdots, l_{n}\right)\left(g,\left(\left(u_{0}, \cdots, u_{m}\right),\left(v_{0}, \cdots, v_{n}\right)\right)\right) \\
=\left(\left(\rho u_{0}, \cdots, \rho u_{m}\right),\left(\rho^{l_{0}} v_{0}, \cdots, \rho^{l_{n}} v_{n}\right)\right),
\end{array}
$$

where $\rho=\exp (2 \pi i / p)$ and $g$ is a generator of $Z_{p}$. The map $f: S^{2 m+1} \times$ $S^{2 n+1} \rightarrow S^{2 m+1} \times S^{2 n+1}$ with

$$
\begin{aligned}
& f\left(\left(u_{0}, \cdots, u_{m}\right),\left(v_{0}, \cdots, v_{n}\right)\right)=\left(\left(u_{0}, \cdots, u_{m}\right), \frac{1}{r}\left(v_{0}^{l_{0}}, \cdots, v_{n}^{l_{n}}\right)\right), \\
& r=\sqrt{\left|v_{0}\right|^{2 l_{0}}+\cdots+\left|v_{n}\right|^{2 l_{n}}},
\end{aligned}
$$

induces a map of orbit spaces:

$$
\tilde{f}_{p}:\left(S^{2 m+1} \times S^{2 n+1}\right) / \phi_{p}(1, \cdots, 1) \rightarrow\left(S^{2 m+1} \times S^{2 n+1}\right) / \phi_{p}\left(l_{0}, \cdots, l_{n}\right) .
$$

Let $\pi:\left(S^{2 m+1} \times S^{2 n+1}\right) / \phi_{p}\left(l_{0}, \cdots, l_{n}\right) \rightarrow\left(S^{2 m+1} \times S^{2 n+1}\right) / \phi\left(l_{0}, \cdots, l_{n}\right) \quad$ be the natural projection. We take up a differentiable embedding

$$
h:\left(S^{2 m+1} \times S^{2 n+1}\right) / \phi(1, \cdots, 1) \rightarrow S^{2 N}
$$

for a sufficiently large $N$.

Proposition 4.1. In the following commutative diagram

$$
\begin{gathered}
\left(S^{2 m+1} \times S^{2 n+1}\right) / \phi_{p}(1, \cdots, 1) \stackrel{\pi}{\longrightarrow}\left(S^{2 m+1} \times S^{2 n+1}\right) / \phi(1, \cdots, 1) \\
\downarrow \tilde{f}_{p} \times h \pi \\
\left(S^{2 m+1} \times S^{2 n+1}\right) / \phi_{p}\left(l_{0}, \cdots, l_{n}\right) \times S^{2 N} \stackrel{\pi \times i d}{\longrightarrow}\left(S^{2 m+1} \times S^{2 n+1}\right) / \phi\left(l_{0}, \cdots, l_{n}\right) \times S^{2 N} \\
\text { (1) } \tilde{f}_{p} \times h \pi \text { and } \tilde{f} \times h \text { are embeddings } \\
\text { (2) } \pi \times i d \text { is transverse regular to }(\tilde{f} \times h)\left(\left(S^{2 m+1} \times S^{2 n+1}\right) / \phi(1,\right.
\end{gathered}
$$


$\cdots, 1))$

(3)

$$
\begin{array}{r}
(\pi \times i d)^{-1}(\tilde{f} \times h)\left(\left(S^{2 m+1} \times S^{2 n+1}\right) / \phi(1, \cdots, 1)\right) \\
=\left(\tilde{f}_{p} \times h \pi\right)\left(\left(S^{2 m+1} \times S^{2 n+1}\right) / \phi_{p}(1, \cdots, 1)\right) .
\end{array}
$$

Proof. A tangent vector at a point of $\left(S^{2 m+1} \times S^{2 n+1}\right) / \phi_{p}(1, \cdots, 1)$ is described as $\vec{v}+\vec{w}$ with $\vec{v} \in$ the tangent space along the base space of the smooth fiber bundle $\pi:\left(S^{2 m+1} \times S^{2 n+1}\right) / \phi_{p}(1, \cdots, 1) \rightarrow\left(S^{2 m+1} \times S^{2 n+1}\right) /$ $\phi(1, \cdots, 1)\}$ and $\vec{w} \in\{$ the tangent space along the fiber $\}$. Let $d\left(\tilde{f}_{p}\right.$ $\times h \pi)(\vec{v}+\vec{w})=0$, then $d(\tilde{f} \times h)(\vec{v})=0$. Since $\tilde{f} \times h$ is an embedding, $\vec{v}=0$. On the other hand, $d \tilde{f}_{p}$ is injective on each tangent space along the fiber, and $\vec{w}=0$. This implies that $\tilde{f}_{p} \times h \pi$ is embedding, because $\tilde{f}_{p} \times h \pi$ is injective. The differentiable fibration $\pi \times i d$ is transverse regular to any submanifold of $\left(S^{2 m+1} \times S^{2 n+1}\right) / \phi\left(l_{0}, \cdots, l_{n}\right) \times S^{2 N}$.

Q.E.D.

Considering the geometric interpretation of the cobordism group [19], we can see that Proposition 4.1 implies

Proposition 4.2. The induced homomorphism $(\pi \times i d)^{*}: M U^{*}$ $\left(\left(S^{2 m+1} \times S^{2 n+1}\right) / \phi\left(l_{0}, \cdots, l_{n}\right) \times S^{2 N}\right) \rightarrow M U^{*}\left(\left(S^{2 m+1} \times S^{2 n+1}\right) / \phi_{p}\left(l_{0}, \cdots, l_{n}\right) \times S^{2 N}\right)$ sends $D_{M U}^{-1}\left[\left(S^{2 m+1} \times S^{2 n+1}\right) / \phi(1, \cdots, 1), \tilde{f} \times h\right]$ to $D_{M U}^{-1}\left[\left(S^{2 m+1} \times S^{2 n+1}\right) / \phi_{p}(1\right.$, $\left.\cdots, 1), \tilde{f}_{p} \times h \pi\right]$.

Let $\psi_{p}\left(l_{0}, \cdots, l_{n}\right): Z_{p} \times S^{2 n+1} \rightarrow S^{2 n+1}$ be an action of $Z_{p}$ on $S^{2 n+1}$ defined by

$$
\psi_{p}\left(l_{0}, \cdots, l_{n}\right)\left(g,\left(v_{0}, \cdots, v_{n}\right)\right)=\left(\rho^{l_{0}} v_{0}, \cdots, \rho^{l_{n}} v_{n}\right) .
$$

We have a complex line bundle $\hat{\xi}(L): S^{2 n+1} \times{ }_{z_{p}} C^{1} \rightarrow S^{2 n+1} / \psi_{p}\left(l_{0}, \cdots, l_{n}\right)$ by taking the orbit space of an action of $Z_{p}$ on $S^{2 n+1} \times C^{1}$

$$
g \cdot\left(\left(u_{0}, \cdots, u_{n}\right), z\right)=\left(\left(\rho^{l_{0}} u_{0}, \cdots, \rho^{l_{n}} u_{n}\right), \rho z\right)
$$

where $g$ is a generator of $Z_{p}$. Denote by

$$
\tilde{\xi}(L): S^{2 n+1} \times{ }_{z_{p}} C^{1} \rightarrow S^{2 n+1} / Z_{p}
$$

a line bundle over a standard lens space which is the orbit space of an action of $Z_{p}$ on $S^{2 n+1} \times C^{1}$ defined by $g \cdot\left(\left(u_{0}, \cdots, u_{n}\right), \approx\right)=\left(\left(\rho u u_{0}, \cdots, \rho u_{n}\right)\right.$, $\rho z)$. The bordism class of $\tilde{f}_{p} \times \tilde{h}:\left(S^{2 m+1} \times S^{2 n+1}\right) / \phi_{p}(1, \cdots, 1) \rightarrow\left(S^{2 m+1} \times\right.$ 
$\left.S^{2 n+1}\right) / \phi_{p}\left(l_{0}, \cdots, l_{n}\right) \times S^{2 N}$ with the embedding $\tilde{h}$ for a large $N$ is described as follows.

Proposition 4.3. Suppose that $m \leqq n$. Then

$$
\begin{aligned}
& {\left[\left(S^{2 m+1} \times S^{2 n+1}\right) / \phi_{p}(1, \cdots, 1), \tilde{f}_{p} \times \tilde{h}\right]} \\
& \quad=D_{M U}\left\{\pi ^ { * } \left\{\left\langle l_{0}\right\rangle_{F}\left(c_{U}^{1}(\tilde{\xi}(L)) \cdots\left\langle l_{n}\right\rangle_{F}\left(c_{U}^{1}(\tilde{\xi}(L))\right\}\right\} \times\left[P \subset S^{2 N}\right],\right.\right.
\end{aligned}
$$

in $M U_{*}\left(\left(S^{2 m+1} \times S^{2 n+1}\right) / \phi_{p}\left(l_{0}, \cdots, l_{n}\right)\right)$, where $P=\{a$ point $\}$ and $\pi:\left(S^{2 m+1}\right.$ $\left.\times S^{2 n+1}\right) / \phi_{p}\left(l_{0}, \cdots, l_{n}\right) \rightarrow S^{2 m+1} / \psi_{p}(1, \cdots, 1)$ is the natural projection.

Proof. Theorem 3.3 and Proposition 4.2 imply that

$$
\begin{aligned}
& {\left[\left(S^{2 m+1} \times S^{2 n+1}\right) / \phi_{p}(1, \cdots, 1), \tilde{f}_{p} \times h \pi\right]} \\
& \quad=D_{M U}\left\{\pi^{*}\left\{\left\langle l_{0}\right\rangle_{F}\left(c_{U}^{1}(\hat{\xi}(L))\right) \cdots\left\langle l_{n}\right\rangle_{F}\left(c_{U}^{1}(\tilde{\xi}(L))\right)\right\}\right\} \times\left[P \subset S^{2 N}\right] .
\end{aligned}
$$

But $h \pi$ is homotopic to $\tilde{h}$, and the bordism class is homotopy invariant, and hence the proposition follows.

The map $f: S^{2 n+1} \rightarrow S^{2 n+1}$ with $f\left(v_{0}, \cdots, v_{n}\right)=\frac{1}{r}\left(v_{0}^{l_{0}}, \cdots, v_{n}^{l_{n}}\right), r$ the norm of $\left(v_{0}^{l_{0}}, \cdots, v_{n}^{l_{n}}\right)$, induces a map of orbit spaces

$$
\widehat{f}_{p}: S^{2 n+1} / \psi_{p}(1, \cdots, 1) \rightarrow S^{2 n+1} / \psi_{p}\left(l_{0}, \cdots, l_{n}\right) .
$$

Theorem 4. 4. In $M U_{*}\left(S^{2 n+1} / \psi_{p}\left(l_{0}, \cdots, l_{n}\right)\right),\left[S^{2 n+1} / \psi_{p}(1, \cdots, 1), \widehat{f}_{p}\right]$ $=D_{M U}\left\{\left\langle l_{0}\right\rangle_{F}\left(c_{U}^{1}(\hat{\xi}(L))\right) \cdots\left\langle l_{n}\right\rangle_{F}\left(c_{U}^{1}(\hat{\xi}(L))\right)\right\}$.

Proof. Define $\pi_{2}:\left(S^{2 n+1} \times S^{2 n+1}\right) / \phi_{p}\left(l_{0}, \cdots, l_{n}\right) \rightarrow S^{2 n+1} / \psi_{p}\left(l_{0}, \cdots, l_{n}\right)$ by $\pi_{2}[u, v]=[v]$ and take a differentiable embedding $h: S^{2 n+1} / \psi_{p}\left(l_{0}, \cdots, l_{n}\right) \rightarrow$ $S^{2 N}$ for a sufficiently large $N$. In the commutative diagram

$$
\begin{gathered}
\left(S^{2 n+1} \times S^{2 n+1}\right) / \phi_{p}(1, \cdots, 1) \stackrel{\pi_{2}}{\longrightarrow} S^{2 n+1} / \phi_{p}(1, \cdots, 1) \\
\downarrow \tilde{f}_{p} \times h \pi_{2} \\
\left(S^{2 n+1} \times S^{2 n+1}\right) / \phi_{p}\left(l_{0}, \cdots, l_{n}\right) \times S^{2 N} \stackrel{\pi_{2} \times i d}{\longrightarrow} S^{2 n+1} / \hat{f}_{p}\left(l_{0}, \cdots, l_{n}\right) \times S^{2 N}
\end{gathered}
$$

$\tilde{f}_{p} \times h \pi_{2}$ is an embedding and $\pi_{2} \times i d$ is transverse regular to $\left(\tilde{f}_{p} \times h\right)$ $\left(S^{2 n+1} / \psi_{p}(1, \cdots, 1)\right)$. Thus it follows that 


$$
\begin{aligned}
& \left(\pi_{2} \times i d\right)^{*} D_{M U}^{-1}\left[S^{2 n+1} / \psi_{p}(1, \cdots, 1), \widehat{f}_{p} \times h\right] \\
& \quad=D_{M U}^{-1}\left[\left(S^{2 n+1} \times S^{2 n+1}\right) / \phi_{p}(1, \cdots, 1), \tilde{f}_{p} \times h \pi_{2}\right] .
\end{aligned}
$$

We now note that the induced bundle $\pi^{!} \tilde{\xi}(L)$ by the projection $\pi$ : $\left(S^{2 n+1}\right.$ $\left.\times S^{2 n+1}\right) / \phi_{p}\left(l_{0}, \cdots, l_{n}\right) \rightarrow S^{2 n+1} / \psi_{p}(1, \cdots, 1)$ is isomorphic to the induced bundle $\pi_{2}: \hat{\xi}(L)$ by the natural projection $\pi_{2}:\left(S^{2 n+1} \times S^{2 n+1}\right) / \phi_{p}\left(l_{0}, \cdots, l_{n}\right)$ $\rightarrow S^{2 n+1} / \psi_{p}\left(l_{0}, \cdots, l_{n}\right)$. Proposition 4.3 implies that

$$
\begin{aligned}
& \left(\pi_{2} \times i d\right) * D_{M U}^{-1}\left(\left[S^{2 n+1} / \psi_{p}(1, \cdots, 1), \widehat{f}_{p} \times h\right]\right) \\
& \quad=\pi_{2}^{*}\left\{\left\langle l_{0}\right\rangle_{F}\left(c_{U}^{1}(\hat{\xi}(L))\right) \cdots\left\langle l_{n}\right\rangle_{F}\left(c_{U}^{1}(\hat{\xi}(L))\right)\right\} \times D_{M U}^{-1}\left[P \subset S^{2 N}\right] .
\end{aligned}
$$

Since $\left(\pi_{2} \times i d\right)^{*}$ is injective, it follows that

$$
\begin{aligned}
& {\left[S^{2 n+1} / \psi_{p}(1, \cdots, 1), \widehat{f}_{p} \times h\right]} \\
& \quad=D_{M U}\left\{\left\langle l_{0}\right\rangle_{F}\left(c_{U}^{1}(\hat{\xi}(L))\right) \cdots\left\langle l_{n}\right\rangle_{F}\left(c_{U}^{1}(\hat{\xi}(L))\right)\right\} \times\left[P \subset S^{2 N}\right] .
\end{aligned}
$$

Applying the homomorphism $M U_{*}\left(S^{2 n+1} / \psi_{p}\left(l_{0}, \cdots, l_{n}\right) \times S^{2 N}\right) \rightarrow M U_{*}\left(S^{2 n+1} /\right.$ $\left.\psi_{p}\left(l_{0}, \cdots, l_{n}\right)\right)$ induced by the projection, we obtain the assertion.

Theorem 4.5. Let $\hat{g}_{p}: S^{2 n+1} / \psi_{p}\left(l_{0}, \cdots, l_{n}\right) \rightarrow S^{2 n+1} / \psi_{p}(1, \cdots, 1) \quad b e$ the map of orbit spaces defined by

$$
\widehat{g}_{p}\left[v_{0}, \cdots, v_{n}\right]=\left[\frac{1}{r}\left(v_{0}^{l_{0}^{\prime}}, \cdots, v_{n}^{l_{n}^{\prime}}\right)\right]
$$

where $l_{j} l_{j}^{\prime} \equiv 1$ modulo $p$ and $r$ is the norm of $\left(v_{0}^{l_{0}^{\prime}}, \cdots, v_{n}^{l_{n}^{\prime}}\right)$. Then

$$
\begin{aligned}
D_{M U}^{-1} & {\left[S^{2 n+1} / \psi_{p}\left(l_{0}, \cdots, l_{n}\right), \widehat{g}_{p}\right] } \\
& \equiv\left\langle l_{0}^{\prime}\right\rangle_{F}\left(\left[l_{0}\right]_{F}(x)\right) \cdots\left\langle l_{n}^{\prime}\right\rangle_{F}\left(\left[l_{n}\right]_{F}(x)\right) \quad \text { modulo }\left(\langle p\rangle_{F}(x)\right)
\end{aligned}
$$

where $\langle p\rangle_{F}(x) \in M U^{*}\left(S^{2 n+1} / \psi_{p}(1, \cdots, 1)\right)$ and $x=c_{U}^{1}(\tilde{\xi}(L))$.

Proof. Consider the natural injection $j: S^{2 n+1} / \psi_{p}(1, \cdots, 1) \rightarrow S^{2 n+3} /$ $\psi_{p}(1, \cdots, 1)$. We can see that $j \widehat{g}_{p} \widehat{f}_{p} \simeq j$ and $\widehat{g}_{p}^{!}(\widetilde{\xi}(L)) \cong \widehat{\xi}(L)$. We note that the Atiyah-Poincare isomorphism $D_{M U}: M U^{*}(X) \rightarrow M U_{*}(X), X$ a weakly almost complex manifold, is given by

$$
D_{M U}(z)=z \cap[X, \text { identity }] \text {. }
$$

We put $U=\left[S^{2 n+1} / \psi_{p}(1, \cdots, 1)\right.$, identity $] \in M U_{2 n+1}\left(S^{2 n+1} / \psi_{p}(1, \cdots, 1)\right)$ and $\widetilde{U}=\left[S^{2 n+1} / \psi_{p}\left(l_{0}, \cdots, l_{n}\right)\right.$, identity $] \in M U_{2 n+1}\left(S^{2 n+1} / \psi_{p}\left(l_{0}, \cdots, l_{n}\right)\right)$. Let 
us compute with Theorem 4.4

$$
\begin{aligned}
j_{*}(U) & =j_{*} \widehat{g}_{p *} \widehat{f}_{p *}(U) \\
& =j_{*} \widehat{g}_{p *}\left\{\left[S^{2 n+1} / \psi_{p}(1, \cdots, 1), \widehat{f}_{p}\right]\right\} \\
& =j_{*} \widehat{g}_{p *}\left\{\widehat{g}_{p}^{*}\left\{\left\langle l_{0}\right\rangle_{F}(x) \cdots\left\langle l_{n}\right\rangle_{F}(x)\right\} \cap \widetilde{U}\right\} \\
& =j_{*}\left\{\left\langle l_{0}\right\rangle_{F}(x) \cdots\left\langle l_{n}\right\rangle_{F}(x) \cap \widehat{g}_{p *}(\widetilde{U})\right\} \\
& =j_{*}\left\{\left\{\left\langle l_{0}\right\rangle_{F}(x) \cdots\left\langle l_{n}\right\rangle_{F}(x) \cup D_{M U}^{-1}\left(\widehat{g}_{p *}(\widetilde{U})\right)\right\} \cap U\right\} .
\end{aligned}
$$

Hence $\left\langle l_{0}\right\rangle_{F}(x) \cdots\left\langle l_{n}\right\rangle_{F}(x) \cup D_{M U}^{-1}\left(\widehat{g}_{p *}(\widetilde{U})\right)-1$ belongs to $D_{M U}^{-1}\left(j_{*}^{-1}(0)\right)$. We recall the following commutative diagram:

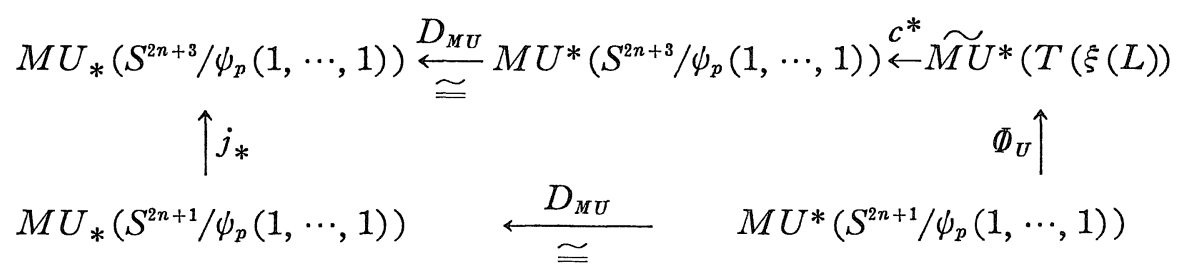

where $\Phi_{U}$ is the Thom isomorphism and $c$ is the canonical collapsing map. Since $\Phi_{U}^{-1} c^{*-1}(0)$ is generated by $\langle p\rangle_{F}(x)$ (cf. [12]), $\left\langle l_{0}\right\rangle_{F}(x) \cdots$ $\left\langle l_{n}\right\rangle_{F}(x) \cup D_{M U}^{-1}\left(\widehat{g}_{p *}(\widetilde{U})\right)-1$ belongs to the ideal generated by $\langle p\rangle_{F}(x)$ in $M U^{*}\left(S^{2 n+1} / \psi_{p}(1, \cdots, 1)\right)$. On the other hand, since $\left\{\tilde{\xi}(L)^{l_{j}}\right\}^{l_{j}^{\prime}} \cong \tilde{\xi}(L)$, we get

$$
\left\{\left\langle l_{j}^{\prime}\right\rangle_{F}\left(\left[l_{j}\right]_{F}(x)\right)\right\}\left[l_{j}\right]_{F}(x)=x
$$

and it follows from Lemma 5 of [9] that $\left\{\left\langle l_{j}^{\prime}\right\rangle_{F}\left(\left[l_{j}\right]_{F}(x)\right)\right\}\left\langle l_{j}\right\rangle_{F}(x)-1$ belongs to an ideal generated by $\langle p\rangle_{F}(x)$. Then we have

$$
\begin{aligned}
D_{M U}^{-1} \widehat{g}_{p *}(\widetilde{U}) & \\
\equiv & \left\{\left\langle l_{0}\right\rangle_{F}(x) \cdots\left\langle l_{n}\right\rangle_{F}(x)\right\}\left\{\left\langle l_{0}^{\prime}\right\rangle_{F}\left(\left[l_{0}\right]_{F}(x)\right) \cdots\right. \\
& \left.\quad\left\langle l_{n}^{\prime}\right\rangle_{F}\left(\left[l_{n}\right]_{F}(x)\right) \cup D_{M U}^{-1}\left(\widehat{g}_{p *}(\widetilde{U})\right)\right\} \quad \text { modulo }\left(\langle p\rangle_{F}(x)\right),
\end{aligned}
$$

and

$$
\begin{array}{r}
D_{M U}^{-1}\left(\widehat{g}_{p *}(\widetilde{U})\right) \equiv\left\langle l_{0}^{\prime}\right\rangle_{F}\left(\left[l_{0}\right]_{F}(x)\right) \cdots\left\langle l_{0}^{\prime}\right\rangle_{F}\left(\left[l_{n}\right]_{F}(x)\right) \text { modulo }\left(\langle p\rangle_{F}(x)\right) . \\
\text { Q.E.D. }
\end{array}
$$

Let us consider the composite 


$$
j_{*}: M U_{*}\left(S^{2 n+1} / \psi_{p}(1, \cdots, 1)\right) \stackrel{i_{*}}{\longrightarrow} M U_{*}\left(B Z_{p}\right) \stackrel{\vartheta}{\cong} M U_{*}\left(Z_{p}\right)
$$

where $i_{*}$ is the $M U_{*}$-homomorphism induced from the natural injection and $\vartheta$ is the natural isomorphism given in [5]. Now we shall prove the Kasparov theorem.

Theorem 4. 6. Assume that $l_{j} l_{j}^{\prime} \equiv 1$ modulo $p$. Then

$$
\begin{aligned}
{\left[S^{2 n+1},\right.} & \left.\psi_{p}\left(l_{0}, \cdots, l_{n}\right)\right] \\
\quad & j_{*} D_{M U}\left\{\left\langle l_{0}^{\prime}\right\rangle_{F}\left(\left[l_{0}\right]_{F}(x)\right) \cdots\left\langle l_{n}^{\prime}\right\rangle_{F}\left(\left[l_{n}\right]_{F}(x)\right)\right\},
\end{aligned}
$$

where $x=c_{U}^{1}(\tilde{\xi}(L)) \in M U^{2}\left(S^{2 n+1} / \psi_{p}(1, \cdots, 1)\right)$.

Proof. From Theorem 4.5 there exists $h(x) \in M U^{2}\left(S^{2 n+1} / \psi_{p}(1, \cdots\right.$, 1)) such that

$$
\begin{aligned}
D_{M U}^{-1}[ & {\left[S^{2 n+1} / \psi_{p}\left(l_{0}, \cdots, l_{n}\right), \widehat{g}_{p}\right] } \\
& =\left\langle l_{0}^{\prime}\right\rangle_{F}\left(\left[l_{0}\right]_{F}(x)\right) \cdots\left\langle l_{n}^{\prime}\right\rangle_{F}\left(\left[l_{n}\right]_{F}(x)\right)+\langle p\rangle_{F}(x) h(x)
\end{aligned}
$$

and

$$
\begin{aligned}
& {\left[S^{2 n+1} / \psi_{p}\left(l_{0}, \cdots, l_{n}\right), \widehat{g}_{p}\right]} \\
& \quad=\left\{\left\langle l_{0}^{\prime}\right\rangle_{F}\left(\left[l_{0}\right]_{F}(x)\right) \cdots\left\langle l_{n}^{\prime}\right\rangle_{F}\left(\left[l_{n}\right]_{F}(x)\right)+\langle p\rangle_{F}(x) h(x)\right\} \cap U
\end{aligned}
$$

where $U=\left[S^{2 n+1} / \psi_{p}(1, \cdots, 1)\right.$, identity $]$. Let $\bar{x}$ be the first cobordism Chern class of the canonical line bundle $\tilde{\xi}(L)$ over $S^{2 n+3} / \psi_{p}(1, \cdots, 1)$ and let

$$
\bar{U}=\left[S^{2 n+3} / \psi_{p}(1, \cdots, 1), \text { identity }\right]
$$

which belongs to $M U_{2 n+3}\left(S^{2 n+3} / \psi_{p}(1, \cdots, 1)\right)$. Then we have

$$
\bar{x} \cap \bar{U}=i_{*} U \quad \text { (cf. [11]). }
$$

Noting that $[p]_{F}(\bar{x})=0$, we calculate

$$
\begin{aligned}
i_{*} & {\left[S^{2 n+1} / \psi_{p}\left(l_{0}, \cdots, l_{n}\right), \widehat{g}_{p}\right] } \\
& =i_{*}\left\{i^{*}\left\{\left\langle l_{0}^{\prime}\right\rangle_{F}\left(\left[l_{0}\right]_{F}(\bar{x})\right) \cdots\left\langle l_{n}^{\prime}\right\rangle_{F}\left(\left[l_{n}\right]_{F}(\bar{x})\right)+\langle p\rangle_{F}(\bar{x}) h(\bar{x})\right\} \cap U\right\} \\
& =\left\{\left\langle l_{0}^{\prime}\right\rangle_{F}\left(\left[l_{0}\right]_{F}(\bar{x})\right) \cdots\left\langle l_{n}^{\prime}\right\rangle_{F}\left(\left[l_{n}\right]_{F}(\bar{x})\right)+\langle p\rangle_{F}(\bar{x}) h(\bar{x})\right\} \cap i_{*}(U) \\
& =\left\langle l_{0}^{\prime}\right\rangle_{F}\left(\left[l_{0}\right]_{F}(\bar{x})\right) \cdots\left\langle l_{n}^{\prime}\right\rangle_{F}\left(\left[l_{n}\right]_{F}(\bar{x})\right) \cap i_{*}(U)
\end{aligned}
$$




$$
=i_{*}\left\{D_{M U}\left(\left\langle l_{0}^{\prime}\right\rangle_{F}\left(\left[l_{0}\right]_{F}(x)\right) \cdots\left\langle l_{n}^{\prime}\right\rangle_{F}\left(\left[l_{n}\right]_{F}(x)\right)\right\}\right.
$$

Q.E.D.

\section{§. Characteristic Classes of $\xi\left(V_{a}\right)$}

The product space $I \times X$ of a $Z_{p}$-space $X$ and an interval $I=[0,1]$ has a $Z_{p}$-action with $g \cdot(t, x)=(t, g \cdot x)$, and we have $Z_{p}$-spaces

$$
\begin{aligned}
& S(X): \text { the usual suspension of } X \\
& C^{+}(X)=X \times[1 / 2,1] / X \times\{1\} \\
& C^{-}(X)=X \times[0,1 / 2] / X \times\{0\} .
\end{aligned}
$$

Denote by $p_{0}$ and $p_{1}$ vertices obtained by the identification of $X \times 0$ and $X \times 1$ in these spaces. A map $\varepsilon_{i}: E Z_{p} \times_{z_{p}}\{p\} \rightarrow E Z_{p} \times_{z_{p}} S(X)$ is defined to be $\varepsilon_{i}(x, p)=\left(x, p_{i}\right)$, and a map $\pi: E Z_{p} \times_{z_{p}} X \rightarrow E Z_{p} \times_{z_{p}}\{p\}=B Z_{p}$ is defined to be $\pi(y, x)=(y, p)$. We can derive the following propositions after the fashion of Proposition 10.1 and Theorem 10.2 of [4].

Proposition 5.1. Suppose that $X$ is a compact $Z_{p}$-space. Then there exists an exact sequence:

$$
M U^{*}\left(E Z_{p} \times_{z_{p}} S(X)\right) \stackrel{\varepsilon_{1}^{*}-\varepsilon_{0}^{*}}{\longrightarrow} M U^{*}\left(B Z_{p}\right) \stackrel{\pi^{*}}{\longrightarrow} M U^{*}\left(E Z_{p} \times_{z_{p}} X\right) .
$$

Proof. $\widetilde{M U}^{*}\left(\left(E Z_{p}\right)^{+} \wedge z_{p}-\right)$ is an equivariant cohomology theory described in [10]. Consider the Mayer-Vietoris exact sequence for a triple $\left(\{S(X)\}^{+} ;\left\{C^{+}(X)\right\}^{+},\left\{C^{-}(X)\right\}^{+}\right)$

$$
\begin{gathered}
\longrightarrow M U^{*}\left(E Z_{p} \times_{z_{p}} S(X)\right) \stackrel{j^{*}}{\longrightarrow} M U^{*}\left(E Z_{p} \times_{z_{p}} C^{+}(X)\right) \\
\oplus M U^{*}\left(E Z_{p} \times_{z_{p}} C^{-}(X)\right) \\
\stackrel{k^{*}}{\longrightarrow} M U^{*}\left(E Z_{p} \times_{z_{p}} X\right) \longrightarrow
\end{gathered}
$$

where $j^{*}(x)=\left(j_{1}^{*}(x), j_{0}^{*}(x)\right)$ and $k^{*}\left(x_{1}, x_{0}\right)=i_{1}^{*}\left(x_{1}\right)-i_{0}^{*}\left(x_{0}\right)$, and $j_{s}$ and $i_{s}$ are natural inclusions. The isomorphisms $M U^{*}\left(E Z_{p} \times{ }_{z_{p}} C^{+}(X)\right) \cong M U^{*}$ $\left(B Z_{p}\right)$ and $M U^{*}\left(E Z_{p} \times_{z_{p}} C^{-}(X)\right) \cong M U^{*}\left(B Z_{p}\right)$ yield the proposition. 
Let $\Psi: \operatorname{Vect}_{C}(-) \rightarrow M U^{*}(-)$ be a natural transformation assigning a complex vector bundle over $X$ to an element of $M U^{*}(X)$ which satisfies

$$
\Psi\left(f^{\prime} \zeta\right)=f^{*} \Psi(\zeta)
$$

Consider complex vector bundles

$$
\xi\left(V_{a}\right) ; E Z_{p} \times_{z_{p}} V_{a} \rightarrow B Z_{p}
$$

where $V_{a}$ is the complex $Z_{p}$-module obtained by the tangent space at an isolated fixed point $a$ of an almost complex $Z_{p}$-manifold $M$. Then we have

Proposition 5.2. Suppose that $a$ and $b$ are isolated fixed points of a simply connected almost complex $Z_{p}$-manifold. If $H^{i}\left(B Z_{p}\right.$; $\left.\left\{\pi_{i}(M)\right\}\right) \cong 0$ for $1 \leqq i \leqq 2 n-1$, then $\Psi\left(\xi\left(V_{a}\right)\right)-\Psi\left(\xi\left(V_{b}\right)\right)$ belongs to an ideal generated by $x^{n}$ in $M U^{*}\left(B Z_{p}\right) \cong M U^{*}[[x]] /\left([p]_{F}(x)\right)$, where $x=c_{U}^{1}(\xi(L))$, L the canonical one dimensional complex $Z_{p}$-module.

Proof. The $(2 n-1)$-skeleton of $E Z_{p}$ can be taken to be $S^{2 n-1}$ with the action given by the complex $n$-dimensional $Z_{p}$-module $n L$. We take

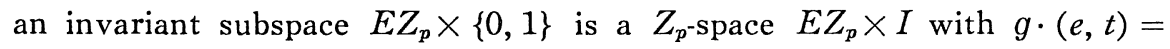
$(g \cdot e, t)$. Consider the constant maps

$$
h_{0}: E Z_{p} \rightarrow\{b\} \quad \text { and } \quad h_{1}: E Z_{p} \rightarrow\{a\}
$$

which induce maps

$$
\tilde{h}_{0}: S^{2 n-1} \subset E Z_{p} \rightarrow\{b\} \text { and } \tilde{h}_{1}: S^{2 n-1} \subset E Z_{p} \rightarrow\{a\} .
$$

We can construct an equivariant homotopy $h: S^{2 n-1} \times I \rightarrow M$ between $\tilde{h}_{0}$ and $\tilde{h}_{1}$, by using the condition for the colomology $H^{i}\left(B Z_{p} ;\left\{\pi_{i}(M)\right\}\right)$, and an equivariant map $\tilde{h}: S\left(S^{2 n-1}\right) \rightarrow M$ (cf. [4, p. 355]). Since

$$
\xi\left(V_{a}\right)=\varepsilon_{i}^{!}\left(i d \times{ }_{z_{p}} \tilde{h}\right) ! \tilde{\tau} \text { and } \xi\left(V_{b}\right)=\varepsilon_{0}^{!}\left(i d \times{ }_{z_{p}} \tilde{h}\right) ! \tilde{\tau},
$$

where $\tilde{\tau}$ denotes a vector bundle $E Z_{p} \times{ }_{z_{p}} E(\tau(M)) \rightarrow E Z_{p} \times_{z_{p}} M$, it follows from Proposition 5.1 that $\pi^{*}\left(\Psi\left(\xi\left(V_{a}\right)\right)-\Psi\left(\xi\left(V_{b}\right)\right)\right)=0$. By using the Gysin exact sequence

$$
\rightarrow M U^{*}\left(B Z_{p}\right) \stackrel{\cdot x^{n}}{\longrightarrow} M U^{*+2 n}\left(B Z_{p}\right) \stackrel{\pi^{*}}{\longrightarrow} M U^{*+2 n}\left(E Z_{p} \times_{z_{p}} S^{2 n-1}\right) \rightarrow
$$

we complete the proof. 
We consider the symmetric polynomial $P_{\omega}\left(\mathfrak{S}_{1}, \cdots, \mathfrak{S}_{n}\right)$ discussed in Section 3, and put $c_{\omega}^{U}\left(\gamma_{n}\right)=P_{\omega}\left(c_{U}^{1}\left(\gamma_{n}\right), \cdots, c_{U}^{n}\left(\gamma_{n}\right)\right)$, where $c_{U}^{i}\left(\gamma_{n}\right)$ is the $i$-th cobordism Chern class [7]. The Landweber-Novikov operation

$$
S_{\omega}^{U}: M U^{*}(X) \rightarrow M U^{*+2|\omega|}(X)
$$

is defined as follows: for $x=[f], f: S^{2 n-k} X^{+} \rightarrow M U(n)$,

$$
S_{\omega}^{U}(x)=\sigma^{k-2 n} f^{*} \Phi_{U}\left(c_{\omega}^{U}\left(\gamma_{n}\right)\right) \quad(\text { cf. [14], [17]). }
$$

The Boardman map $\beta_{U^{r}}: M U^{*}(X) \rightarrow(M U \wedge M U)^{*}(X) \cong M U^{*}(X)\left[\left[t_{1}\right.\right.$, $\left.\left.t_{2}, \cdots\right]\right]$ is defined by

$$
\beta_{U}(x)=\sum_{\omega} S_{\omega}^{U}(x) t^{\omega} \quad(\mathrm{cf} .[2],[19]),
$$

which is natural and multiplicative. Let $J(G)$ be the set of isomorphism classes of non trivial irreducible complex $Z_{p}$-modules, and let $C=\left\{V_{j_{1}}^{k_{1}} \oplus\right.$ $\cdots \oplus V_{j_{l}}^{k_{l}} \mid V_{j_{s}} \in J(G)$ and $k$ 's are non negative integers $\}$. We consider the multiplicative system $S$ consisting of cobordism Euler classes $\left\{e\left(E Z_{p} \times_{z_{p}}\right.\right.$

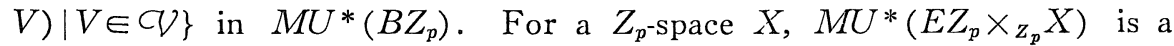
$M U^{*}\left(B Z_{p}\right)$-module by a map $E Z_{p} \times_{z_{p}} X \rightarrow B Z_{p} \times\left(E Z_{p} \times_{z_{p}} X\right)$ sending [e, $x]$ to $([e],[e, x])$. The localized module $S^{-1} M U^{*}\left(E Z_{p} \times_{z_{p}} X\right)$ of the $M U^{*}\left(B Z_{p}\right)$-module $M U^{*}\left(E Z_{p} \times_{z_{p}} X\right)$ consists of all fractions $\{x / e ; x \in$ $\left.M U^{*}\left(E Z_{p} \times_{z_{p}} X\right), e \in S\right\}$. For a complex vector bundle $\zeta$ over $X$, we put

$$
c_{t}^{U}(\zeta)=1+\sum_{\omega} c_{\omega}^{U}(\zeta) t^{\omega}
$$

which is an invertible element of $M U^{*}\left[\left[t_{1}, t_{2}, \cdots\right]\right]$. We define $\tilde{\beta}_{U}$ : $S^{-1} M U^{*}\left(E Z_{p} \times_{z_{p}} X\right) \rightarrow S^{-1} M U^{*}\left(E Z_{p} \times{ }_{z_{p}} X\right)\left[\left[t_{1}, t_{2}, \cdots\right]\right]$ by

$$
\tilde{\beta}_{U}(y / e(\xi(V)))=\left(\beta_{U}(y) \cdot \frac{1}{c_{t}^{U}(\xi(V))}\right) / c(\xi(V))
$$

which is multiplicative and natural. Moreover, we define

$$
\widetilde{S}_{\omega}^{U}: S^{-1} M U^{*}\left(E Z_{p} \times_{z_{p}} X\right) \rightarrow S^{-1} M U^{*}\left(E Z_{p} \times_{z_{p}} X\right)
$$

by $\tilde{\beta}_{U}(x / e)=\sum_{\omega} \widetilde{S}_{\omega}^{U}(x / e) t^{\omega}$.

Proposition 5. 3. The operation $\widetilde{S}_{\omega}^{U}$ on $S^{-1} M U^{*}\left(E Z_{p} \times_{z_{p}}-\right)$ have the following properties:

(1) $\widetilde{S}_{\omega}^{U}$ is natural.

(2) $\widetilde{S}_{\omega}^{U}\left(\left(x_{1} / e_{1}\right) \cdot\left(x_{2} / e_{2}\right)\right)=\sum_{\omega=\left(\omega^{\prime} \omega^{\prime \prime}\right)} \widetilde{S}_{\omega^{\prime}}^{U}\left(x_{1} / e_{1}\right) \widetilde{S}_{\omega^{\prime \prime}}^{U}\left(x_{2} / e_{2}\right)$, where for 


$$
\begin{aligned}
& \omega^{\prime}=\left(j_{1}^{\prime}, \cdots, j_{s}^{\prime}\right) \text { and } \omega^{\prime \prime}=\left(j_{1}^{\prime \prime}, \cdots, j_{t}^{\prime \prime}\right),\left(\omega^{\prime} \omega^{\prime \prime}\right) \text { denotes }\left(j_{1}^{\prime}, \cdots,\right. \\
& \left.j_{s}^{\prime}, j_{1}^{\prime \prime}, \cdots, j_{t}^{\prime \prime}\right) \text {. }
\end{aligned}
$$

(3) $\widetilde{S}_{\omega}^{U}(x / 1)=S_{\omega}^{U}(x) / 1$, where $S_{\omega}^{U}$ is the ordinary LandweberNovikov operation, i.e. $\lambda S_{\omega}^{U}=\widetilde{S}_{\omega}^{U} \lambda$, where $\lambda: M U^{*}\left(E Z_{p} \times_{z_{p}}-\right)$ $\rightarrow S^{-1} M U^{*}\left(E Z_{p} \times_{z_{p}}-\right)$ is the canonical map.

(4) For $\omega=(\underbrace{1, \cdots, 1}_{i_{1}}, \underbrace{2, \cdots, 2}_{i_{2}}, \cdots, \underbrace{k, \cdots, k}_{i_{k}})$,

$$
\widetilde{S}_{\omega}^{U}(1 / e(\xi(L)))=(-1)^{i_{1}+\cdots+i_{k}}\left\{\frac{\left(i_{1}+\cdots+i_{k}\right) !}{i_{1} ! i_{2} ! \cdots i_{k} !} e(\xi(L))^{|\omega|-1}\right\} / 1 .
$$

Proof. By making use of the multiplicativity and the naturality of $\beta_{U}$, we derive (1) and (2). For a zero dimensional complex $Z_{p}$-module 0 , we have $e(\xi(0))=1$ and $c_{t}^{U}(\xi(0))=1$, and

$$
\begin{aligned}
\tilde{\beta}_{U}(x / 1) & =\beta_{U}(x) \cdot \frac{1}{c_{t}^{U}(\xi(0))} / e(\xi(0)) \\
& =\beta_{U}(x) / 1
\end{aligned}
$$

which implies (3). To prove (4), we calculate

$$
\begin{aligned}
\tilde{\beta}_{U}(1 / e(\xi(L))) \\
\quad=\left\{\beta_{U}(1) \cdot \frac{1}{c_{t}^{U}(\xi(L))}\right\} / e(\xi(L)) \\
\quad=\left\{\frac{1}{1+e(\xi(L)) t_{1}+e(\xi(L))^{2} t_{2}+\cdots}\right\} / e(\xi(L)) \\
=\left\{\sum(-1)^{i}\left(e(\xi(L)) t_{1}+e(\xi(L))^{2} t_{2}+\cdots\right)^{i}\right\} / e(\xi(L)) .
\end{aligned}
$$

This completes the proof.

We see easily the following

Proposition 5.4. $S_{\omega}^{U}(e(\xi(V)))=e(\xi(V)) c_{\omega}^{U}(\xi(V))$.

Taking two complex $Z_{p}$-modules $V_{a}$ and $V_{b}$ obtained from tangent spaces at isolated fixed points $a$ and $b$ of an almost complex $Z_{p}$-manifold, a fraction $e\left(\xi\left(V_{a}\right)\right) / e\left(\xi\left(V_{b}\right)\right)$ is an integral element from the following 
proposition.

Proposition 5.5. Suppose that $L$ is a canonical complex one dimensional $Z_{p}$-module. Take $k_{i}$ and $l_{j}$ such that $\left(k_{i}, p\right)=1$ and $\left(l_{j}\right.$. $p)=1$. Then for $n \geqq m, e\left(\xi\left(L^{k_{1}} \oplus \cdots \oplus L^{k_{n}}\right)\right) / e\left(\xi\left(L^{l_{1}} \oplus \cdots \oplus L^{l_{m}}\right)\right)$ belongs to the image of $\lambda: M U^{*}\left(B Z_{p}\right) \rightarrow S^{-1} M U^{*}\left(B Z_{p}\right)$ which sends $x$ to $x / 1$.

Proof. For $x=c_{U}^{1}(\xi(L))$,

$$
e\left(\xi\left(L^{k}\right)\right)=[k]_{F}(x)=k x+a_{1}^{(k)} x^{2}+a_{2}^{(k)} x^{3}+\cdots
$$

and

$$
e\left(\xi\left(L^{k}\right)\right) / x=\langle k\rangle_{F}(x) / 1 .
$$

Assume that $(l, p)=1$, then there is an integer $l^{\prime}$ such that $l^{\prime} l \equiv 1$ modulo $p$ and

$$
x=\left\langle l^{\prime}\right\rangle_{F}\left([l]_{F}(x)\right) \cdot[l]_{F}(x) .
$$

Therefore we have

$$
\begin{aligned}
& \frac{e\left(\xi\left(L^{k_{1}} \oplus \cdots \oplus L^{k_{n}}\right)\right)}{e\left(\xi\left(L^{l_{1}} \oplus \cdots \oplus L^{l_{m}}\right)\right)} \\
& =\left\langle l_{1}^{\prime}\right\rangle_{F}\left(\left[l_{1}\right]_{F}(x)\right) \cdots\left\langle l_{m}^{\prime}\right\rangle_{F}\left(\left[l_{m}\right]_{F}(x)\right)\left\langle k_{1}\right\rangle_{F}(x) \cdots \\
& \quad\left\langle k_{m}\right\rangle_{F}(x)\left[k_{m+1}\right](x) \cdots\left[k_{n}\right]_{F}(x) / 1 .
\end{aligned}
$$

where $l_{j}^{\prime} l_{j} \equiv 1$ module $p$.

Q.E.D.

Proof of Theorem $A$. For brevity, we put $e_{a}=e\left(\xi\left(V_{a}\right)\right)$ and $e_{b}$ $=e\left(\xi\left(V_{b}\right)\right)$. We show by induction with respect to the length of the partition $\omega$ that

$$
\widetilde{S}_{\omega}^{U}\left(\frac{e_{a}}{e_{b}}\right)=\frac{e_{a}}{e_{b}} \cdot \frac{h_{\omega}(x) \cdot x^{n}}{1}
$$

where $h_{\omega}(x) \in M U^{*}\left(B Z_{p}\right)$. By using (2) of Proposition 5.3 we obtain

$$
\widetilde{S}_{(i)}^{U}\left(\frac{e_{a}}{1}\right)=\widetilde{S}_{(i)}^{U}\left(\frac{e_{a}}{e_{b}}\right) \cdot \frac{e_{b}}{1}+\frac{e_{a}}{c_{b}} \cdot \widetilde{S}_{(i)}^{U}\left(\frac{e_{b}}{1}\right) .
$$

Hence it follows from (3) of Propositions 5.3 and 5.4 that 


$$
\widetilde{S}_{(i)}^{U}\left(\frac{e_{a}}{e_{b}}\right)=\frac{e_{a}}{e_{b}} \cdot \frac{c_{(i)}^{U}\left(\xi\left(V_{a}\right)\right)-c_{(i)}^{U}\left(\xi\left(V_{b}\right)\right)}{1} .
$$

Proposition 5. 2 implies that there is an element $h_{(i)}(x) \in M U^{*}\left(B Z_{p}\right)$ such that $c_{(i)}^{U}\left(\xi\left(V_{a}\right)\right)-c_{(i)}^{U}\left(\xi\left(V_{b}\right)\right)=h_{(i)}(x) x^{n}$, and

$$
\widetilde{S}_{(i)}^{U}\left(\frac{e_{a}}{e_{b}}\right)=\frac{e_{a}}{e_{b}} \cdot \frac{h_{(i)}(x) x^{n}}{1} .
$$

Suppose the result is proved for $\omega^{\prime}$ whose length is less than the length of $\omega$. By using (2) of Proposition 5.3 with the inductive hypothesis we calculate

$$
\begin{aligned}
\widetilde{S}_{\omega}^{U}\left(\frac{e_{a}}{1}\right)=\widetilde{S}_{\omega}^{U}\left(\frac{e_{a}}{e_{b}} \cdot \frac{e_{b}}{1}\right) \\
=\widetilde{S}_{\omega}^{U}\left(\frac{e_{a}}{c_{b}}\right) \cdot \frac{e_{b}}{1}+\widetilde{S}_{\omega}^{U}\left(\frac{e_{b}}{1}\right) \cdot \frac{e_{a}}{e_{b}}+\sum_{\omega=\left(\omega^{\prime} \omega^{\prime \prime}\right)} \frac{e_{a}}{e_{b}} \cdot \frac{h_{\omega^{\prime}}(x) x^{n} S_{\omega^{\prime \prime}}^{U}\left(e_{b}\right)}{1}
\end{aligned}
$$

where $h_{\omega^{\prime}}(x) \in M U^{*}\left(B Z_{p}\right)$. Moreover it follows from Propositions 5. 4 and 5.2 that there exists an element $\tilde{h}_{\omega}(x) \in M U^{*}\left(B Z_{p}\right)$ such that

$$
\widetilde{S}_{\omega}^{U}\left(\frac{e_{a}}{e_{b}}\right)=\frac{e_{a}}{e_{b}} \tilde{h}_{\omega}(x) x^{n} / 1-\sum_{\omega=\left(\omega^{\prime} \omega^{\prime \prime}\right)} \frac{e_{a}}{e_{b}}\left\{h_{\omega^{\prime}}(x) x^{n} c_{\omega^{\prime \prime}}^{U}\left(\xi\left(V_{b}\right)\right)\right\} / 1,
$$

and there is an element $h_{\omega}(x) \in M U^{*}\left(B Z_{p}\right)$ such that

$$
\tilde{S}_{\omega}^{U}\left(\frac{e_{a}}{e_{b}}\right)=\frac{e_{a}}{e_{b}} h_{\omega}(x) x^{n} / 1
$$

It is pointed out by [9] that the canonical map $\lambda: M U^{*}\left(B Z_{p}\right) \rightarrow S^{-1} M U^{*}$ $\left(B Z_{p}\right)$ with $\lambda(x)=x / 1$ has the kernel which is an ideal generated by $\langle p\rangle_{F}(x)$. We then complete the proof.

\section{$\S 6$. On the Bordism Classes of Actions on Invariant Spheres around the Isolated Fixed Points}

The Thom homomorphism $\mu: M U^{*}(-) \rightarrow H^{*}(-)$ is the multiplicative natural transformation with the following properties.

Proposition 6. 1. Let $\zeta$ be a complex vector bundle over $X$. Then 
(1) $\mu c_{\omega}^{U}(\zeta)=c_{\omega}^{H}(\zeta)$

(2) $\mu \Phi_{U}(x)=\Phi(\mu(x))$, where $\Phi_{U}: M U^{*}(X) \rightarrow \widetilde{M U} *(T(\zeta))$ and $\Phi: H^{*}(X) \rightarrow \widetilde{H}^{*}(T(\zeta))$ are the Thom homomorphisms.

Recall the following property of the Umkehr homomorphism [8].

Proposition 6. 2. $g_{!}\left(g^{*}(x) \cup y\right)=x \cup g_{!}(y)$.

We observe $S_{\omega}^{H}: M U^{*}(X) \rightarrow H^{*}(X)$ for a weakly complex manifold $X$.

Proposition 6. 3. Take an element $x=[M \stackrel{g}{\rightarrow} X] \in M U_{*}(X)$, where $X$ is a weakly complex manifold and $g$ is a differentiable map. Then,

$$
S_{\omega}^{H} D_{M U}^{-1}(x)=\sum_{\omega=\left(\omega^{\prime} \omega^{\prime \prime}\right)} c_{\omega^{\prime}}^{H}(\widetilde{\tau}(X)) g_{!}\left(c_{\omega^{\prime \prime}}^{H}(\nu)\right)
$$

where $\nu$ is the normal bundle of $M$ in a Euclidean space with the complex structure and $\tilde{\tau}(X)$ is the Whitney sum of $\tau(X)$ and some trivial bundle which is a complex bundle.

Proof. Let $\tilde{g}: M \rightarrow X \times R^{l}$ be an embedding with the normal bundle $\widetilde{\nu}$ equipped with a complex structure and $\widetilde{g} \simeq g . D_{M U}^{-1}(x)$ is represented by the composition

$$
S^{\imath} \wedge X^{+} \stackrel{c}{\longrightarrow} T(\widetilde{\nu}) \stackrel{\widehat{g}}{\longrightarrow} M U(k)
$$

which $c$ is the collapsing map and $\hat{g}$ is the map induced by the classifying map for $\nu$. The Whitney sum $\widetilde{\nu} \oplus \tau(M)$ is stably equivalent to $g ! \tau(X)$ and

$$
c_{t}^{H}(\widetilde{\mathcal{V}}) \cdot c_{t}^{H}(\widetilde{\tau}(M))=g^{*} c_{t}^{H}(\widetilde{\tau}(X)) .
$$

Hence we have that $c_{t}^{H}(\widetilde{\mathcal{\nu}})=g^{*} c_{t}^{H}(\widetilde{\tau}(X)) \cdot c_{t}^{H}(\nu)$. We calculate with Propositions 6.1 and 6.2

$$
\begin{aligned}
S_{\omega}^{H} D_{M U}^{-1}(x) & =\mu S_{\omega}^{U} D_{M U}^{-1}(x)=\sigma^{-\imath} c^{*}\left\{\Phi\left(c_{\omega}^{H}(\widetilde{\mathcal{\nu}})\right)\right\} \\
& =g_{!}\left(c_{\omega}^{H}(\widetilde{\mathcal{\nu}})\right) \\
& =g_{!}\left(\sum_{\omega=\left(\omega^{\prime} \omega^{\prime \prime}\right)} g^{*}\left(c_{\omega^{\prime}}^{H}(\widetilde{\tau}(X)) c_{\omega^{\prime \prime}}^{H}(\nu)\right)\right)
\end{aligned}
$$




$$
=\sum_{\omega=\left(\omega^{\prime} \omega^{\prime \prime}\right)} c_{\omega^{\prime}}^{H}(\widetilde{\tau}(X)) g_{!}\left(c_{\omega^{\prime \prime}}^{H}(\nu)\right) .
$$

Q.E.D.

$M U^{k}$ is isomorphic to $M U_{-k}$ and a bordism class [M] of a weakly almost complex manifold can be regarded to be in $M U^{*}$. Directly Proposition 6.3 implies

Corollary 6. 4. $\mu S_{\omega}^{U}[M]=\left\langle c_{\omega}^{H}(\nu),[M]\right\rangle$, where $\nu$ is the normal vector bundle of $M$ in a Euclidean space which is equipped with the complex structure, where $c_{\left(i_{1}, \ldots, i_{l}\right)}^{H}$ is the Chern class for $\sum t_{1}^{i_{1}} \cdots t_{l}^{i_{l}}$.

We consider the ideal $\mathcal{I}_{p}$ in $M U^{*}$ which is generated by $p, a_{1}^{(p)}$, $a_{2}^{(p)}, \cdots, a_{k}^{(p)}, \cdots$ which are coefficients of

$$
[p]_{F}(x)=p x+a_{1}^{(p)} x^{2}+a_{2}^{(p)} x^{3}+\cdots .
$$

We recall the following property of $\mathcal{G}_{p}$.

Proposition 6.5 (cf. [9]). [M] belongs to $\mathcal{G}_{p}$ if and only if $c_{\omega}^{H}[M]=\left\langle c_{\omega}^{H}(\tau(M)),[M]\right\rangle \equiv 0$ modulo $p$, for any $\omega$, where $p$ is prime.

Proof. Let $y=c_{U}^{1}(\eta)$ be the cobordism first Chern class of the Hopf bundle $\eta$ over $C P^{\infty}$. It is known (cf. [14], [17]) that

$$
S_{\omega}^{U}\left([p]_{F}(y)\right)=\left\{\begin{array}{l}
\left\{[p]_{F}(y)\right\}^{i+1} \text { if } \omega=(i) \\
0 \quad \text { otherwise. }
\end{array}\right.
$$

We see $S_{\omega}^{H}\left([p]_{F}(y)\right) \equiv 0$ modulo $p$, and

$$
S_{\omega}^{H}\left(p y+a_{1}^{(p)} y^{2}+a_{2}^{(p)} y^{3}+\cdots\right) \equiv 0 \text { modulo } p .
$$

Then we can deduce that $S_{\omega}^{H}\left(a_{i}^{(p)}\right) \equiv 0$ modulo $p$. Therefore we have that the Chern numbers of $[N]$ are zero modulo $p$ if $[N]$ belongs to $\mathcal{I}_{p}$. The Hopf bundle $\tilde{\eta}$ over $C P^{n}$ satisfies that

$D_{M U}\left(c_{U}^{1}\left(\tilde{\eta}^{q}\right)\right)=q\left[C P^{n-1} \subset C P^{n}\right]+a_{1}^{(q)}\left[C P^{n-2} \subset C P^{n}\right]+\cdots+a_{n-1}^{(q)}[P \subset$ $\left.C P^{n}\right]$, in $M U_{*}\left(C P^{n}\right)$. Let $D_{M U}\left(c_{U}^{1}\left(\tilde{\eta}^{q}\right)\right)=\left[V_{(q)}^{n-1} \subset C P^{n}\right]$, then

(*) $\left[V_{(q)}^{n-1}\right]=q\left[C P^{n-1}\right]+a_{1}^{(q)}\left[C P^{n-2}\right]+\cdots+a_{n-1}^{(q)}$.

We note that $V_{(q)}^{n-1}$ is a $U$-submanifold dual to $c_{H}^{1}\left(\tilde{\eta}^{q}\right)$ (cf. $[7$, p. 81]), 
and the fundamental classes of $V_{(q)}^{n-1}$ and $C P^{n}$ satisfy that $i_{*}\left[V_{(q)}^{n-1}\right]=c_{H}^{1}\left(\tilde{\eta}^{q}\right)$ $\cap\left[C P^{n}\right], i: V_{(q)}^{n-1} \subset C P^{n}$. Noting that the normal bundle $\nu$ of $V_{(q)}^{n-1}$ in $C P^{n}$ is isomorphic to $i ! \tilde{\eta}^{q}$, we have that $c_{(n-1)}^{H}\left(\tau\left(V_{(q)}^{n-1}\right)\right)=i^{*}\left\{(n+1)-q^{n-1}\right\}$ $\widetilde{y}^{n-1}$, where $\tilde{y}=c_{H}^{1}(\tilde{\eta})$. Therefore it follows that the Chern number $c_{(n-1)}^{H}$ $\left[V_{(q)}^{n-1}\right]=q(n+1)-q^{n}$. Using $(*)$ and $c_{(n-1)}^{H}\left[C P^{n-1}\right]=n$, we have $c_{(n-1)}^{H}$ $\left[a_{n-1}^{(q)}\right]=q-q^{n}$. For prime $q$, we take

$$
\left[W_{q^{k-1}}\right]=a_{q^{k-1}}^{(q)}+q^{b}\left[C P^{u}\right], b=q^{k}-k \text { and } u=q^{k}-1
$$

whose Chern number $c_{\left(q^{k}-1\right)}^{H}\left[W_{q^{k-1}}\right]$ equals to $q$. Take a $2 i$-dimensional weakly almost complex manifold $W_{i}, i \neq q^{k}-1$ for any prime $q$, such that $c_{(i)}^{H}\left[W_{i}\right]=1$. According to $[16], M U^{*}=Z\left[\left[W_{1}\right],\left[w_{2}\right], \cdots\right]$. Assume that $c_{\omega}^{H}[M] \equiv 0$ modulo $p$ for any $\omega$ and

Noting that

$$
[M]=\sum a_{i_{1} \cdots i_{n}}\left[W_{1}\right]^{i_{1}} \ldots\left[W_{n}\right]^{i_{n}} .
$$

$$
\begin{aligned}
& S_{\underbrace{H}_{i_{1}}, \cdots, 1,2, \cdots, 2}^{H} \underbrace{H}_{i_{2}}, \underbrace{\left.n_{1}, \cdots, n\right)}_{i_{n}}\left[W_{1}\right]^{i_{1}}\left[W_{2}\right]^{i_{2}} \cdots\left[W_{n}\right]^{i_{n}} \\
& =\left(c_{(1)}^{H}\left[W_{1}\right]\right)^{i_{1}}\left(c_{(2)}^{H}\left[W_{2}\right]\right)^{i_{2}} \cdots\left(c_{(n)}^{H}\left[W_{n}\right]\right)^{i_{n}},
\end{aligned}
$$

we inductively deduce that if $i_{s}=0$ for $s=p^{k}-1$, then $a_{i_{1} i_{2} \cdots i_{n}} \equiv 0$ modulo $p$, and $[M] \in \mathcal{I}_{p}$.

Q.E.D.

We now go back to consider the cobordism Euler class of complex vector bundle $\xi\left(V_{a}\right): E Z_{p} \times_{z_{p}} V_{a} \rightarrow B Z_{p}, V_{a}$ the complex $Z_{p}$-module given by the tangent space at the isolated fixed points of a $Z_{p}$-manifold.

Proposition 6.6. Suppose that $V_{a}$ and $V_{b}$ are complex $Z_{p}$-modules given by tangent spaces at isolated fixed points $a$ and $b$ of $a$ simply connected almost complex $Z_{p}$-manifold $M$, and $\lambda(\alpha)=e\left(\xi\left(V_{a}\right)\right) /$ $e\left(\xi\left(V_{b}\right)\right)$, where $\lambda: M U^{*}\left(B Z_{p}\right) \rightarrow S^{-1} M U^{*}\left(B Z_{p}\right)$ is the canonical homomorphism. If $H^{i}\left(B Z_{p} ;\left\{\pi_{i}(M)\right\}\right) \cong 0$ for $1 \leqq i \leqq 2 n-1$, then

$$
\alpha=\lambda_{0}+\lambda_{1} x+\lambda_{2} x^{2}+\cdots
$$

where $\lambda_{1}, \lambda_{2}, \cdots, \lambda_{n-1}$ belong to $\mathcal{I}_{p}$.

Proof. Suppose that $|\omega|=2 i, 1 \leqq i \leqq n-1$. Then $S_{\omega}^{U} \lambda_{k} \in M U^{2 i-2 k}$. 
Note that $\mu: M U^{k}(P) \rightarrow H^{k}(P), P=\{$ a point $\}$, is the zero homomorphism for $k>0$, and $S_{\omega}^{U}\left(\lambda_{0}\right)=0$ if $\omega \neq(0)$. Suppose that $\lambda_{j}, j=1,2, \cdots, i-1$, belong to $\mathcal{G}_{p}$. Then

$$
\mu S_{\omega}^{U}(\alpha)=\mu S_{\omega}^{U}\left(\lambda_{i}\right) \cdot x_{H}^{i}=c_{\omega}^{H}\left[\lambda_{i}\right] x_{H}^{i},
$$

where $x_{H}=c_{H}^{1}(\xi(L))$. Since $S_{\omega}^{U}(\alpha)$ belongs to an ideal generated by $x^{n}$ and $\langle p\rangle_{F}\left(c_{U}^{1}(\xi(L))\right)$ from Theorem $\mathrm{A}, c_{\omega}^{H}\left[\lambda_{i}\right] x_{H}^{i}=0$ in $H^{*}\left(B Z_{p}\right)$. Proposition 6.5 implies that $\lambda_{i} \in \mathcal{I}_{p}$.

Q.E.D.

Proof of Theorem $B$. Let $\tilde{\xi}(V)$ be a complex vector bundle $S^{2 k-1}$ $\times_{Z_{p}} V \rightarrow S^{2 k-1} / Z_{p}$, where $V$ is a complex $Z_{p}$-module and $S^{2 k-1}$ has the $Z_{p}$-action $\psi_{p}(1, \cdots, 1)$. Let $i: S^{2 k-1} / \psi_{p}(1, \cdots, 1) \rightarrow B Z_{p}$ be the natural injection. Put $x=c_{U}^{1}(\xi(L))$ and $\bar{x}=c_{U}^{1}(\widetilde{\xi}(L))$. Then, $i \xi \xi(L) \cong \widetilde{\xi}(L)$. We see that in $S^{-1} M U^{*}\left(B Z_{p}\right)$,

$$
\begin{aligned}
& l_{1} \cdots l_{k} \frac{x^{k}}{e\left(\xi\left(V_{a}\right)\right)}-m_{1} \cdots m_{k} \frac{x^{k}}{e\left(\xi\left(V_{b}\right)\right)} \\
& \quad=l_{1} \cdots l_{k} \frac{x^{k}}{e\left(\xi\left(V_{a}\right)\right)}-m_{1} \cdots m_{k} \frac{x^{k}}{\boldsymbol{e}\left(\xi\left(V_{a}\right)\right)} \cdot \frac{\boldsymbol{e}\left(\xi\left(V_{a}\right)\right)}{\boldsymbol{e}\left(\xi\left(V_{b}\right)\right)} .
\end{aligned}
$$

On the other hand it follows from Proposition 6.6 that

$$
\begin{gathered}
m_{1} \cdots m_{k}\left\langle l_{1}\right\rangle_{F}(x)\left\langle m_{1}^{\prime}\right\rangle_{F}\left(\left[m_{1}\right]_{F}(x)\right) \cdots\left\langle l_{k}\right\rangle_{F}(x)\left\langle m_{k}^{\prime}\right\rangle_{F}\left(\left[m_{k}\right]_{F}(x)\right) \\
\equiv l_{1} \cdots l_{k}+h(x) x^{n} \quad \text { modulo } \mathcal{G}_{p}
\end{gathered}
$$

where $m_{i} m_{i}^{\prime} \equiv 1$ modulo $p$. Therefore we get

$$
\begin{aligned}
l_{1} \cdots l_{k}\left\langle l_{1}^{\prime}\right\rangle_{F}\left(\left[l_{1}\right]_{F}(x)\right) \cdots\left\langle l_{k}^{\prime}\right\rangle_{F}\left(\left[l_{k}\right]_{F}(x)\right) \\
\quad-m_{1} \cdots m_{k}\left\langle m_{1}^{\prime}\right\rangle_{F}\left(\left[m_{1}\right]_{F}(x)\right) \cdots\left\langle m_{k}^{\prime}\right\rangle_{F}\left(\left[m_{k}\right]_{F}(x)\right) \\
\equiv \tilde{h}(x) x^{n} \text { modulo } \mathcal{I}_{p}, l_{i} l_{i}^{\prime} \equiv 1 \text { modulo } p \text {, where } \tilde{h}(x) \in M U^{*}\left(B Z_{p}\right) .
\end{aligned}
$$

Applying $i^{*}$ to the above, we have

$$
\begin{aligned}
l_{1} \cdots l_{k}\left\langle l_{1}^{\prime}\right\rangle_{F}\left(\left[l_{1}\right]_{F}(\bar{x})\right) \cdots\left\langle l_{k}^{\prime}\right\rangle_{F}\left(\left[l_{k}\right]_{F}(\bar{x})\right) \\
\quad-m_{1} \cdots m_{k}\left\langle m_{1}^{\prime}\right\rangle_{F}\left(\left[m_{1}\right]_{F}(\bar{x})\right) \cdots\left\langle m_{k}^{\prime}\right\rangle_{F}\left(\left[m_{k}\right]_{F}(\bar{x})\right) \\
\equiv \tilde{h}(\bar{x}) \bar{x}^{n} \quad \text { modulo } \mathcal{I}_{p}(\text { cf. }[12]) .
\end{aligned}
$$

Since $j_{*} D_{M U} \bar{x}^{n}=\left[S^{2(k-n)-1}, \phi\right]$ (cf. [11]), Theorems 4.5 and 4.6 imply the theorem. 


\section{$\S 7$. The Isolated Fixed Points of $Z_{3}$-Actions}

In this section we will consider an complex structure preserving smooth $Z_{3}$-action $\left(M^{2 k}, \phi\right)$ on a simply connected closed almost complex manifold $M^{2 k}$. Let $a$ and $b$ be isolated fixed points. We describe the induced actions of $Z_{3}$ on the tangent spaces at $a$ and $b$ as complex $Z_{3^{-}}$ modules

$$
V_{a}=s L^{2} \oplus(k-s) L
$$

and

$$
V_{b}=(s+t) L^{2} \oplus(k-s-t) L
$$

Recall that

$$
\langle 2\rangle_{F}(x)=a_{0}^{(2)}+a_{1}^{(2)} x+a_{2}^{(2)} x^{2}+\cdots, a_{i}^{(2)} \in M U^{-2 i}
$$

and

$$
c_{(n)}^{H}\left(a_{n}^{(2)}\right)=2-2^{n+1} .
$$

In this situation we shall first indicate a lemma which is derived as proof of Theorem B.

Lemma 7.1. Suppose that $H^{i}\left(B Z_{3} ;\left\{\pi_{i}\left(M^{2 k}\right)\right\}\right) \cong 0$ for $1 \leqq i \leqq 2 n$ -1. Then for $1 \leqq j \leqq n-1$

$$
\sum_{i_{1}+\cdots+i_{t}=j} a_{i_{1}}^{(2)} \cdots a_{i_{t}}^{(2)} \quad \text { belong to } \mathcal{I}_{3} .
$$

Proof. In $S^{-1} M U^{*}\left(B Z_{3}\right), M U^{*}\left(B Z_{3}\right) \cong M U^{*}[[x]] /[3]_{F}(x)$, we have

$$
\frac{e\left(V_{a}\right)}{e\left(V_{b}\right)}=\mu_{0}+\mu_{1} x+\cdots+\mu_{k} x^{k}+\cdots, \mu_{1}, \cdots, \mu_{n-1} \in \mathcal{I}_{3}
$$

from Proposition 6.6 and

$$
\begin{aligned}
& \frac{2^{s} x^{k}}{\mathcal{e}\left(V_{a}\right)}-\frac{2^{s+t} x^{k}}{e\left(V_{b}\right)}=\widetilde{\mu}_{1} x+\widetilde{\mu}_{2} x^{2}+\cdots+\widetilde{\mu}_{k} x^{k}+\cdots, \\
& \widetilde{\mu}_{1}, \cdots, \widetilde{\mu}_{n-1} \in \mathcal{I}_{3} .
\end{aligned}
$$

Noting the fact that the kernel of the canonical map $\lambda: M U^{*}\left(B Z_{3}\right) \rightarrow$ 
$S^{-1} M U^{*}\left(B Z_{3}\right)$ is the ideal generated by $\langle 3\rangle_{F^{\prime}}(x)$, we obtain

$$
\begin{aligned}
& 2^{s} x^{k} e\left(V_{b}\right)-2^{s+t} x^{k} e\left(V_{a}\right) \\
& \quad=e\left(V_{a}\right) e\left(V_{b}\right)\left\{\widetilde{\mu}_{1} x+\widetilde{\mu}_{2} x^{2}+\cdots+\widetilde{\mu}_{k} x^{k}+\cdots\right\}
\end{aligned}
$$

and

$$
\begin{aligned}
& 2^{s}\left(\left\{\langle 2\rangle_{F}(x)\right\}^{t}-2^{t}\right) \\
& \quad=\hat{\mu}_{1} x+\hat{\mu}_{2} x^{2}+\cdots+\hat{\mu}_{k} x^{k}+\cdots, \hat{\mu}_{1}, \cdots, \hat{\mu}_{n-1} \in \mathcal{I}_{3} \cdot \quad \text { Q.E.D. }
\end{aligned}
$$

Then we obtain the following

Lemma 7.2. Suppose that $H^{i}\left(B Z_{3} ;\left\{\pi_{i}\left(M^{2 k}\right)\right\}\right) \cong 0$ for $1 \leqq i \leqq 2 n$ -1 . Then, for $1 \leqq m \leqq n-1$ the binomial coefficients $\left(\begin{array}{c}t \\ m\end{array}\right)$ are divisible by 3 .

Proof. We take a partition

$$
\omega=(\underbrace{k, \cdots, k}_{j_{k}}, \cdots, \underbrace{2, \cdots, 2}_{j_{2}}, \underbrace{1, \cdots, 1}_{j_{1}}, \underbrace{0, \cdots, 0}_{j_{0}})
$$

of $k$, where

$$
|\omega|=1 \cdot j_{1}+2 \cdot j_{2}+\cdots+k \cdot j_{k}=k
$$

and

$$
j_{0}+j_{1}+\cdots+j_{k}=t
$$

We define now

$$
\begin{gathered}
\|\omega\|=j_{1}+\cdots+j_{k}, \\
a_{\omega}^{(2)}=\left\{a_{k}^{(2)}\right\}^{j_{k}} \cdots\left\{a_{1}^{(2)}\right\}^{j_{1}}\left\{a_{0}^{(2)}\right\}^{j_{0}}
\end{gathered}
$$

and

$$
\lambda_{\omega}=\frac{t !}{j_{k} ! \cdots j_{2} ! j_{1} ! j_{0} !} .
$$

Then we have the following

$$
\sum_{i_{1}+\cdots+i_{t}=j} a_{i_{1}}^{(2)} \cdots a_{i_{t}}^{(2)}=\sum_{|\omega|=j} \lambda_{\omega} a_{\omega}^{(2)} .
$$

We take up the case $k=1$. Since from Lemma 7.1 $2^{t-1} \ell \cdot a_{1}^{(2)}=\sum_{i_{1}+\cdots+i_{t}=1}$ 
$a_{i_{1}}^{(2)} \cdots a_{i_{t}}^{(2)}$ belongs to $\mathcal{I}_{3}$, and $c_{(1)}^{H}\left(a_{1}^{(2)}\right)=-2, t$ is divisible by 3 . Assume that $m<n$ and $\left(\begin{array}{l}t \\ j\end{array}\right), j=1, \cdots, m-1$, are divisible by 3 . From Lemma $7.1 \sum_{\omega \mid=m} \lambda_{\omega} a_{\omega}^{(2)}$ belongs to $\mathcal{G}_{3}$, and for $\|\omega\| \leqq m-1$

$$
\lambda_{\omega}=\frac{\|\omega\| !}{j_{k} ! \cdots j_{2} ! j_{1} !} \cdot\left(\begin{array}{c}
t \\
\|\omega\|
\end{array}\right) \equiv 0 \quad \text { modulo } 3 \text {. }
$$

By induction we complete the proof.

We shall give some information on isolated fixed points of $Z_{3}$-actions.

Theorem 7.3. Let $a$ and $b$ be isolated fixed points of a complex structure preserving smooth action of $Z_{3}$ on the simply connected closed almost complex manifold $M^{2 k}$. Suppose that

$$
k=\lambda_{u} 3^{u}+\lambda_{u-1} 3^{u-1}+\cdots+\lambda_{1} 3+\lambda_{0}, 0 \leqq \lambda_{j} \leqq 2 \text { and } \lambda_{u} \neq 0
$$

and

$$
H^{i}\left(B Z_{3} ;\left\{\pi_{i}\left(M^{2 k}\right)\right\}\right) \cong 0 \text { for } 1 \leqq i \leqq 2 \cdot 3^{u}+1 .
$$

Then $V_{a}$ is equivalent to $V_{b}$.

Proof. Let $\quad V_{a}=s L^{2} \oplus(k-s) L \quad$ and $\quad V_{b}=(s+t) L^{2} \oplus(k-s-t) L$. Suppose that $t=\lambda_{u}^{\prime} 3^{u}+\lambda_{u-1}^{\prime} 3^{u-1}+\cdots+\lambda_{1}^{\prime} 3+\lambda_{0}^{\prime} \leqq k$. It follows from Lemma 7.2 that

$$
\lambda_{l}^{\prime} \equiv\left(\begin{array}{c}
t \\
3^{2}
\end{array}\right) \equiv 0 \quad \text { modulo } 3
$$

Hence $\lambda_{l}^{\prime}=0$ and $t=0$.

Q.E.D.

Corollary 7.4. Suppose that $Z_{3}$ acts on a simply connected almost complex closed $2 k$-dimensional manifold $M$ as a complex structure preserving deffeomorphism with isolated fixed points only. Let $k=\lambda_{u} 3^{u}+\cdots+\lambda_{1} 3+\lambda_{0}, \quad 0 \leqq \lambda_{j} \leqq 2$, and $\lambda_{u} \neq 0$. If $H^{i}\left(B Z_{3} ;\left\{\pi_{i}(M)\right\}\right) \cong 0$ for $1 \leqq i \leqq 2 \cdot 3^{u}+1$, then the number of fixed points is divisible by $3^{[(k-1) / 2]+1}$.

Proof. Let $n$ be the number of the fixed points. Theorem 7.3 
implies that

$$
n\left[S\left(V_{a}\right), \phi_{a}\right]=0 \quad \text { in } \quad M U_{*}\left(Z_{3}\right)
$$

where $V_{a}=s L^{2}+(k-s) L$. The Kasparov theorem (Theorem 4.6) implies that

$$
n(l+3 m)\left[S^{2 k-1}, \widetilde{\phi}\right]+\mu_{1}\left[S^{2 k-3}, \widetilde{\phi}\right]+\cdots+\mu_{k-1}\left[S^{1}, \widetilde{\phi}\right]=0
$$

where $l \not \equiv 0$ modulo 3 and $\mu_{i} \in \Gamma(3), \Gamma(3)\left[\left[C P^{2}\right]\right]=M U_{*}$ (cf. [6], [11]). From the result of [6] and [11] we can derive the assersion.

\section{References}

[1] Adams, J. F., Stable homotopy and generalised homology, Chicago, Univ. of Chicago Math. Lecture Notes, 1974.

[2] Araki, S., Typical formal group in cobordism and K-theory, Lecture in Math. Kyoto Univ., Kinokuniya, 1973.

[3] Atiyah, M. F., Bordism and cobordism, Proc. Camb. Phil. Soc., 57 (1961), 200-208.

[4] Bredon, G. E., Introduction to compact transformation groups, Academic Press, 1972.

[5] Conner, P. E., and Floyd, E. E., Differentiable periodic maps, Springer, BerlinHeiderberg-New York, 1964.

[6] - Periodic maps which preserve a complex structure, Bull. Amer. Math. Soc., 70 (1964), 574-579.

[7] - The relation of cobordism to K-theories, Lecture Notes in Math., 28, Springer, Berlin-Heiderberg-New York, 1966.

[8] Dyer, E., Cohomology theories, Benjamin, 1969.

[9] Tom Dieck, T., Actions of finite p-groups without stationary points, Topology, 9 (1970), 359-366.

[10] - Lokalisierung äquivarianter Kohomologie-Theorien, Math. Z., 121 (1971), 253-262.

[11] Kamata, M., The structure of the bordism group $U_{*}\left(B Z_{p}\right)$, Osaka J. Math., 7 (1970), 409-416.

[12] Notes on the cobordism group $U^{*}\left(L^{n}(m)\right)$, Osaka J. Math., 9 (1972), 287-292.

[13] Kasparov, G. G., Invariants of classical lens manifolds in cobordism theory, Izv. Akad. Nauk SSSR. Ser. Math., 33 (1969), 753-747, Math., USSR. Izv., 3 (1969), 695-706.

[14] Landweber, P. S., Cobordism operations and Hopf algebras, Trans. Amer. Math. Soc., 129 (1967), 94-110.

[15] - Coherence, flatness and cobordism of classifying spaces, Proc. Adv. Study Inst. Alg. Top., Aarhus (1970), 256-269.

[16] Milnor, J. W., On the cobordism ring $\Omega^{*}$ and a complex analogue, Part I, Amer. J. Math., 82 (1960), 505-521.

[17] Novikov, S. P., The method of algebraic topology from the viewpoint of cobordism theories, Izv. Akad. Nauk SSSR, 31 (1967), Math. USSR Izv., 1 (1967), 827-913.

[18] Ozeki, H. and Uchida, F., Principal circle actions on a product of spheres, Osaka J, Math. 9 (1972), 379-390.

[19] Quillen, D., Elementary proofs of some results of cobordism theory using Steenrod operations, Advances on Math., 7 (1971), 29-56.

[20] Switzer, R. M., Algebraic topology-homotopy and homology, Springer-Verlag, BerlinHeiderberg-New York, 1975. 